\title{
The Mesorhizobium huakuii transcriptional regulator AbiEi plays a critical role in nodulation and is important for bacterial stress response
}

Xiaohong Chen ${ }^{\dagger}$, Aiqi Hu ${ }^{\dagger}$, Qian Zou, Sha Luo, Hetao Wu, Chunlan Yan, Tao Liu, Donglan He, Xiaohua Li and Guojun Cheng*

\begin{abstract}
Background: Bacterial abortive infection (Abi) systems are type IV toxin-antitoxin (TA) system, which could elicit programmed cell death and constitute a native survival strategy of pathogenic bacteria under various stress conditions. However, no rhizobial AbiE family TA system has been reported so far. Here, a M. huakuii AbiE TA system was identified and characterized.

Results: A mutation in M. huakuii abiEi gene, encoding an adjacent GntR-type transcriptional regulator, was generated by homologous recombination. The abiEi mutant strain grew less well in rich TY medium, and displayed increased antioxidative capacity and enhanced gentamicin resistance, indicating the abiEi operon was negatively regulated by the antitoxin AbiEi in response to the oxidative stress and a particular antibiotic. The mRNA expression of abiEi gene was significantly up-regulated during Astragalus sinicus nodule development. The abiEi mutant was severely impaired in its competitive ability in rhizosphere colonization, and was defective in nodulation with $97 \%$ reduction in nitrogen-fixing capacity. The mutant infected nodule cells contained vacuolation and a small number of abnormal bacteroids with senescence character. RNA-seq experiment revealed it had 5 up-regulated and 111 down-regulated genes relative to wild type. Of these down-regulated genes, 21 are related to symbiosis nitrogen fixation and nitrogen mechanism, 16 are involved in the electron transport chain and antioxidant responses, and 12 belong to type VI secretion system (T6SS).
\end{abstract}

Conclusions: M. huakuii AbiEi behaves as a key transcriptional regulator mediating root nodule symbiosis.

Keywords: Type IV toxin-antitoxin system, Mesorhizobium huakuii, Transcriptional regulator AbiEi, Symbiosis nitrogen fixation, RNA-Seq analysis of nodule bacteroids

\footnotetext{
* Correspondence: chengguojun@mail.scuec.edu.cn

${ }^{+}$Xiaohong Chen and Aiqi Hu contributed equally to this work.

Hubei Provincial Engineering and Technology Research Center for Resources and Utilization of Microbiology, College of Life Sciences, South-Central

University for Nationalities, Wuhan 430074, Hubei, China
}

(c) The Author(s). 2021 Open Access This article is licensed under a Creative Commons Attribution 4.0 International License, which permits use, sharing, adaptation, distribution and reproduction in any medium or format, as long as you give appropriate credit to the original author(s) and the source, provide a link to the Creative Commons licence, and indicate if changes were made. The images or other third party material in this article are included in the article's Creative Commons licence, unless indicated otherwise in a credit line to the material. If material is not included in the article's Creative Commons licence and your intended use is not permitted by statutory regulation or exceeds the permitted use, you will need to obtain permission directly from the copyright holder. To view a copy of this licence, visit http://creativecommons.org/licenses/by/4.0/ The Creative Commons Public Domain Dedication waiver (http://creativecommons.org/publicdomain/zero/1.0/) applies to the data made available in this article, unless otherwise stated in a credit line to the data. 


\section{Background}

Toxin-antitoxin (TA) systems are ubiquitous constituents found on plasmids or chromosomes of countless bacteria, archaea, and possibly also unicellular fungi [1]. Bacterial TA systems are composed of bicistronic operons encoding a stable toxin that can harm the host cell and its cognate labile antitoxin, which protects the host from the toxin's deleterious effect [2]. The product of the toxin gene is a protein, while the product of the antitoxin is either a protein or a non-coding RNA [3]. TA systems have been shown to play various physiological roles in the formation of dormancy and persister cells, survival during infection, adaptation to hostile environments, programmed cell death and biofilm formation [4-7]. Based on the biochemical nature and mode of action of the antitoxin gene product, bacterial TA systems have been divided into six types: small regulatory RNAs in types I and III, and antitoxins of proteinaceous nature in type II, IV, V and VI TA systems $[8,9]$.

The proteinaceous antitoxin of type IV system neutralizes its cognate toxin by forming toxin-antitoxin complexes instead of a direct protein-protein interaction [10]. Several common families of type IV system have been identified on the chromosomes of bacteria and archaea: CbtA/CbeA, YkfI/YafW, YpjF/YfjZ, and AbiEii/ AbiEi. The pair CbtA/CbeA was the first type IV system found in E. coli. The toxin CbtA alters cell shape by inhibiting the polymerization of cytoskeletal proteins FtsZ and MreB through direct interaction, without showing direct interaction with its cognate antitoxin [3]. The first AbiE system was reported in Lactococcus lactis where it acts at the post-transcriptional level in the lytic cycle to abort phage development [11]. AbiE was found to be a type IV TA system as there is no apparently direct interaction between the antitoxin and toxin, and the antitoxin antagonizes toxin activity by stabilizing its targets [12]. The AbiEii toxin is a putative nucleotidyltransferase containing a C-terminal domain involved in toxin neutralization. The AbiEi antitoxin, a transcriptional regulator, contains an $\mathrm{N}$-terminal domain required for repression of $a b i E$ transcription, and a bi-functional Cterminal domain required for transcriptional repression and sufficient for toxin neutralization [13]. Previous work on the Streptococcus agalactiae AbiE system revealed that AbiEi negatively autoregulates the abiE promoter and is sufficient for antitoxicity, yet it is unknown whether all systems provide dual resistance functions $[3,13]$.

Bacteria of the family Rhizobiaceae are able to induce symbiotic nodules on the roots of leguminous plants where bacteroids convert atmospheric nitrogen to ammonia [14]. This highly complex process requires a specific signal exchanges between the partners. Compatible rhizobia sense the flavonoids released from their host roots through the transcriptional activator NodD, which trigger nodule organogenesis [15]. Earlier reports described the presence of type II TA modules in Sinorhizobium meliloti and Bradyrhizobium japonicum. The bat/ bto module is classified as a type II TA system belonging to the vapBC-family, and deletion of the B. japonicum bat/bto operon resulted in alterations of several metabolic pathways and defective symbiotic performance due to the changes in lipopolysaccharide (LPS) [16]. Milunovic et al. reported that loss of $4 \mathrm{~S}$. meliloti type II TA systems results in growth inhibition, but does affect the S. meliloti-alfalfa symbiosis [17]. However, the $S$. meliloti $n t r R$ gene, a member of the type II TA system, was controlled negatively by its own product and positively by the symbiotic regulator syrM. Mutation in the $n t r R$ gene induced nodules with enhanced nitrogen fixation capacity [18].

Rhizobial genomes frequently contain type IV AbiE toxin-antitoxin operator, but unlike with the type II TA system, knowledge of regulation of the type IV AbiE system in Rhizobium species is still poorly documented. Here, we identified a type IV TA antitoxin gene $a b i E i$ in M. huakuii 7653R, and the roles of M. huakuii abiEi in free-living bacteria and during $\mathrm{N}_{2}$-fixing symbiosis with A. sinicus were investigated by analyzing the phenotypes of abiEi mutant strain. A transcriptome analysis was also carried out to discover clues that might explain the differences in the nodules induced by the abiEi mutant and the wild type strain. To our knowledge, this work represents the first transcriptome analysis of TA gene in symbiotic root nodules reported to date.

\section{Results}

\section{Determination of AbiE system activity}

In order to elucidate the function of the TA system, the AbiE gene cassette and the toxin gene abiEii was overexpressed in $E$. coli, and the effect of expressed protein product was ruled out by comparing the growth of $E$. coli BL21(DE3) cells in the presence versus the absence of the plasmid (Fig. 1). Under IPTG induction, both $E$. coli strains harboring either recombination vector pETAbiE or pETAbiEii grew more slowly than control cells with the empty vector pET-28a, which indicated that induced expression of $\mathrm{AbiE}$ system or AbiEii toxin protein has a negative effect on the growth of host cells. However, E. coli strains harboring both toxin and antitoxin genes (pETAbiE) grew slightly, but significantly faster than that harboring toxin gene abiEii (pETAbiEii), suggesting antitoxin AbiEi is a transcriptional regulator that can repress abiEii expression to influence toxin.

\section{Construction of $M$. huakuii abiEi mutant}

The gene MCHK_RS33180 in M. huakuii encodes a putative type IV TA system transcriptional regulator AbiEi. The $a b i E i$ gene is predicted to encode a 221-amino acid 


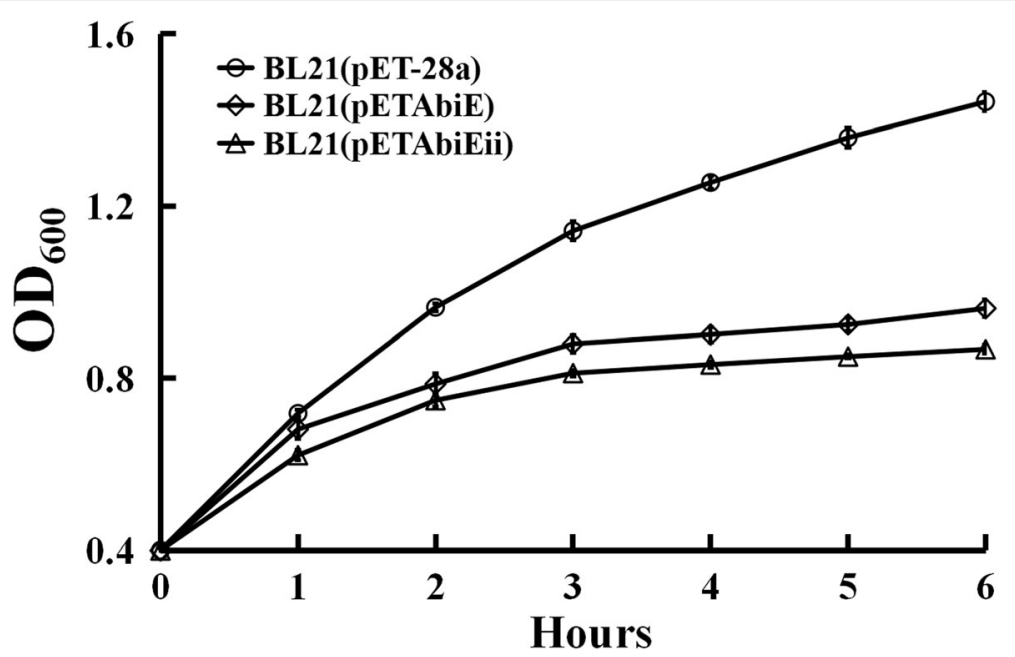

Fig. 1 The growth curve of the E.coli BL21 strains after IPTG induction. E. coli strain BL21(pET-28a), the recombinant strains BL21 (pETAbiE) and BL21 (pETAbiEii) were cultured in $L B$ at $\mathrm{OD}_{600}$ of 0.4 , then induced with $1 \mathrm{mM}$ IPTG. Data are from three biological samples plus and minus the standard error of the mean $( \pm$ SEM)

polypeptide with an expected molecular mass of 24.94 $\mathrm{kDa}$ and a $\mathrm{pI}$ value of 9.95 . To confirm the function of the $a b i E i$ gene in growth, environment stress and symbiotic nitrogen fixation, a mutant HKabiEi strain of this gene was constructed by single crossover homologous recombination. qRT-PCR was firstly employed to examine the relative mRNA levels of antitoxin and toxin in free-living cells from $M$. huakuii. The expression of antitoxin gene abiEi was almost not detected in the mutant HKabiEi, while the expression of toxin gene abiEii was $0.86 \pm 0.11$, and there was no significant difference in the mutant HKabiEi compared to the wild-type strain 7653R. The results confirmed that abiEi was disrupted by the insertion of vector pK19mob in HKabiEi, and also indicated that antitoxin AbiEi didnot directly regulate toxin AbiEi expression, and may antagonize toxin activity by stabilizing its targets. The mutant showed no significant difference in AMS minimal medium with $\mathrm{NH}_{4} \mathrm{Cl}$ as nitrogen source and glucose as carbon source, but exhibited defective growth in rich TY medium (Fig. 2).

\section{Role of the abiEi gene in the regulation of antibiotic resistance}

It has been reported that TA systems constitute a native survival strategy of pathogenic bacteria and thus are potential targets of antibiotic drugs [19]. To examine the possibility that abiEi affects the antibiotic-resistance of M. huakuii, sensitivity to gentamicin (Gm) and chloramphenicol $(\mathrm{Cm})$ at low concentration was assayed in AMS minimal medium. In the presence of $2 \mu \mathrm{g} \mathrm{mL}{ }^{-1} \mathrm{Gm}$, the mutant $\mathrm{HKabiEi}$ was grown to the early logarithmic phase at $24 \mathrm{~h}$, and entered the stationary at $48 \mathrm{~h}$ postinoculation, whereas the parent strain $7653 \mathrm{R}$ was grown to the early logarithmic phase at $48 \mathrm{~h}$, and entered the stationary phase at $72 \mathrm{~h}$ postinoculation (Fig. 3A). The resistance of the $M$. huakuii strain to $2 \mu \mathrm{g} \mathrm{mL}{ }^{-1} \mathrm{Cm}$ stress was also estimated, the $a b i E i$ mutant HKabiEi showed no difference in growth as compared with the wild-type (Fig. 3B). These data indicated that mutantion in $a b i E i$ gene can display different antibiotic susceptibilities of $M$. huakuii.

\section{Role of the abiEi gene in the regulation of redox homeostasis}

It has been reported that the bacterial TA system is a module that plays a role in cell survival under stress conditions [20]. To determine the function of the $M$. huakuii abiEi gene in the regulation of redox homeostasis, the sensitivity of the abiEi mutant strain to the inhibition of growth by $\mathrm{H}_{2} \mathrm{O}_{2}$ or SNP, which could be due to changes in the cellular redox status [21], was examined. The zone of inhibition induced by $\mathrm{H}_{2} \mathrm{O}_{2}$ for mutant HKabiEi significantly decreased as compared with the wild-type 7653R strain (Table 1, Additional file 1). When abiEi on plasmid pBBR1MCS-5 was introduced into mutant $\mathrm{HKabiEi}$, the resulting strain $\mathrm{HKabiEi}(\mathrm{pB}$ BRabiEi) could rescue the variation of the inhibition zone, and showed hypersensitive to $\mathrm{H}_{2} \mathrm{O}_{2}$ as it made significantly bigger $(p<0.05)$ inhibition zone than the wildtype 7653R strain, whereas HKabiEi harboring an empty plasmid showed no significant difference with regard to mutant strain (Table 1, Additional file 1). The resistant to SNP stress was also estimated, and mutant HKabiEi was more resistant to $10 \mu \mathrm{g} \mathrm{mL}^{-1} \mathrm{SNP}$ compared with the wild-type 7653R strain (Fig. 4). These results indicated that $a b i E i$ gene plays a negative regulatory role in the oxidative stress response. 


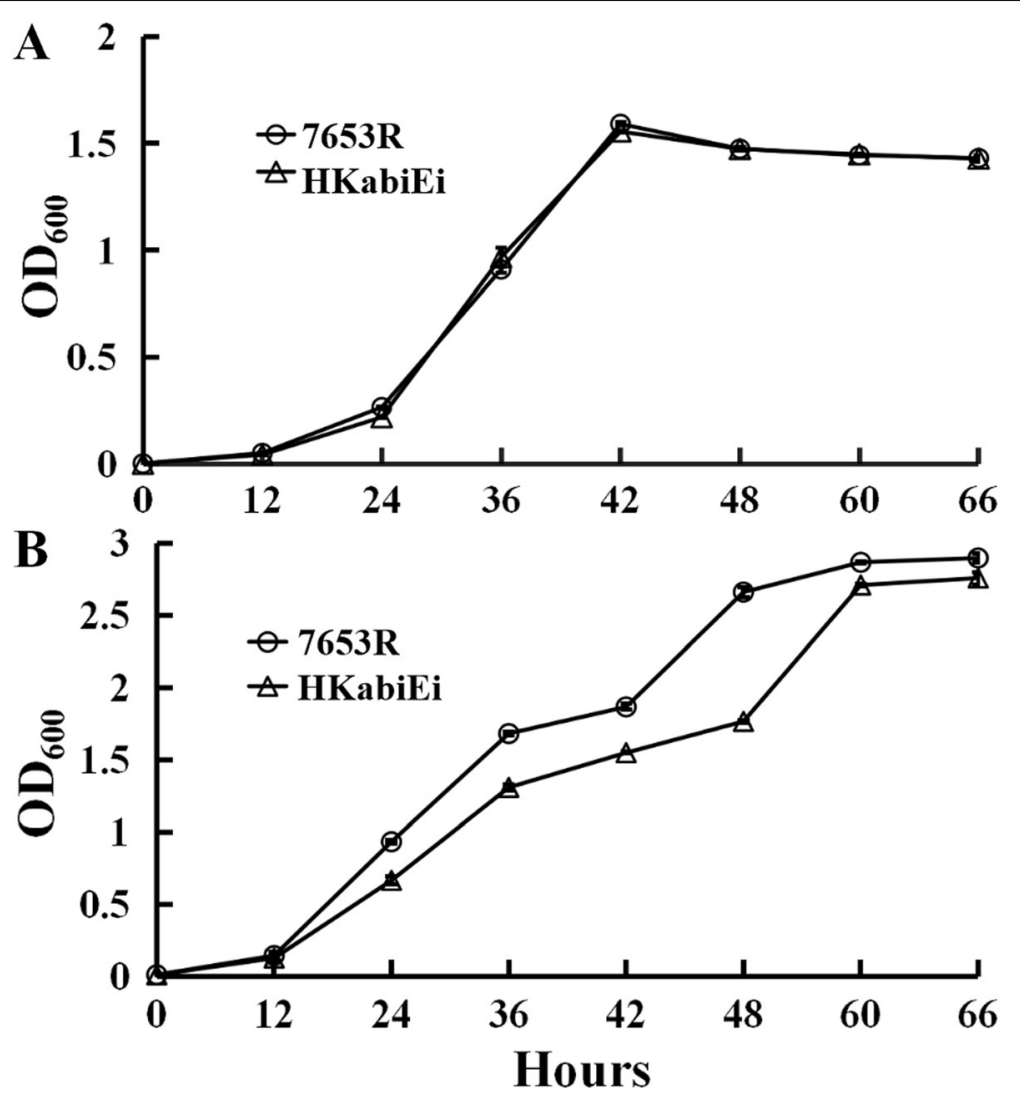

Fig. 2 Growth of M. huakuii in AMS or TY media. Wild-type 7653R and mutant HKabiEi were grown in AMS supplemented with $\mathrm{Glc} / \mathrm{NH} 4^{+}(\mathbf{A})$ and TY (B). Data are from three biological samples plus and minus the standard error of the mean ( \pm SEM)

Effect of mutation of abiEi on rhizosphere competition Competition between the abiEi mutant HKabiEi and parent strain 7653R for growth in the plant rhizosphere was measured by inoculating a low number of $M$. huakuii strains into the $A$. sinicus rhizosphere $\left(10^{3}\right.$ to $10^{4}$ CFU per seedling), and determining the total amount of bacteria after 7 days. When the mutant HKabiEi and the parent 7653R were inoculated alone into short-term colonization of sterile plant rhizosphere, the population density of the mutant strain was a little (but significantly) higher than that of wild-type (Fig. 5). When these strains were inoculated in equal proportions, mutant HKabiEi was at a significant disadvantage $(32.90 \%$ of bacteria recovered). When strain $\mathrm{HKabiEi}$ was inoculated at a 10-fold excess over wild-type 7653R, it still accounted for only $48.68 \%$ of bacteria recovered ( $t$-test; $P \leq 0.01)$. TA systems are ubiquitous bacterial systems that may function in metabolic stress management, but are also thought to play a role in virulence by helping pathogens survive stress [22]. The decreased ability of the abiEi mutant to complete in host plant rhizosphere shows that transcriptional regulator AbiEi is essential for colonization of the host plant rhizosphere by $M$. huakuii.

\section{Effect of mutation of abiEi on nodulation}

In order to assess the nodulation and nitrogen fixing capacity of the abiEi mutant, A. sinicus seedlings were inoculated with $M$. huakuii strains. Four weeks postinoculation, the number, structure, and acetylene reduction activity (ARA) values of the nodules were analyzed (Table 2 and Fig. 6). No statistically significant difference in the number of nodules per plant was observed between plants inoculated with strain $\mathrm{HKabiEi}$ and plants inoculated with wild-type 7653R (Table 2). In contrast, the abiEi mutant HKabiEi elicited small white nodules on $A$. sinicus, and the plants inoculated with the strain HKabiEi were similar to that of plants without inoculation. A notable feature of our study was that the nitrogen fixation capacity was severely affected in the abiEi mutant $\mathrm{HKabiEi}$, with a reduction from $35.20 \mathrm{nmol}$ of $\mathrm{C}_{2} \mathrm{H}_{4}$ plant ${ }^{-1} \mathrm{~h}^{-1}$ in $7653 \mathrm{R}$ to $0.90 \mathrm{nmol}$ of $\mathrm{C}_{2} \mathrm{H}_{4}$ plant $^{-1}$ $\mathrm{h}^{-1}$ in the abiEi mutant (Table 2). Plants nodulated with the complemented strain HKabiEi(pBBRabiEi) had somewhat wild-type properties; approximately the same plant fresh weight, red nodules and reduce acetylene per nodule at the same rate as 7653R-inoculated.

To further investigate the symbiotic role of $a b i E i$ in more detail, sections of mature nodules were prepared 

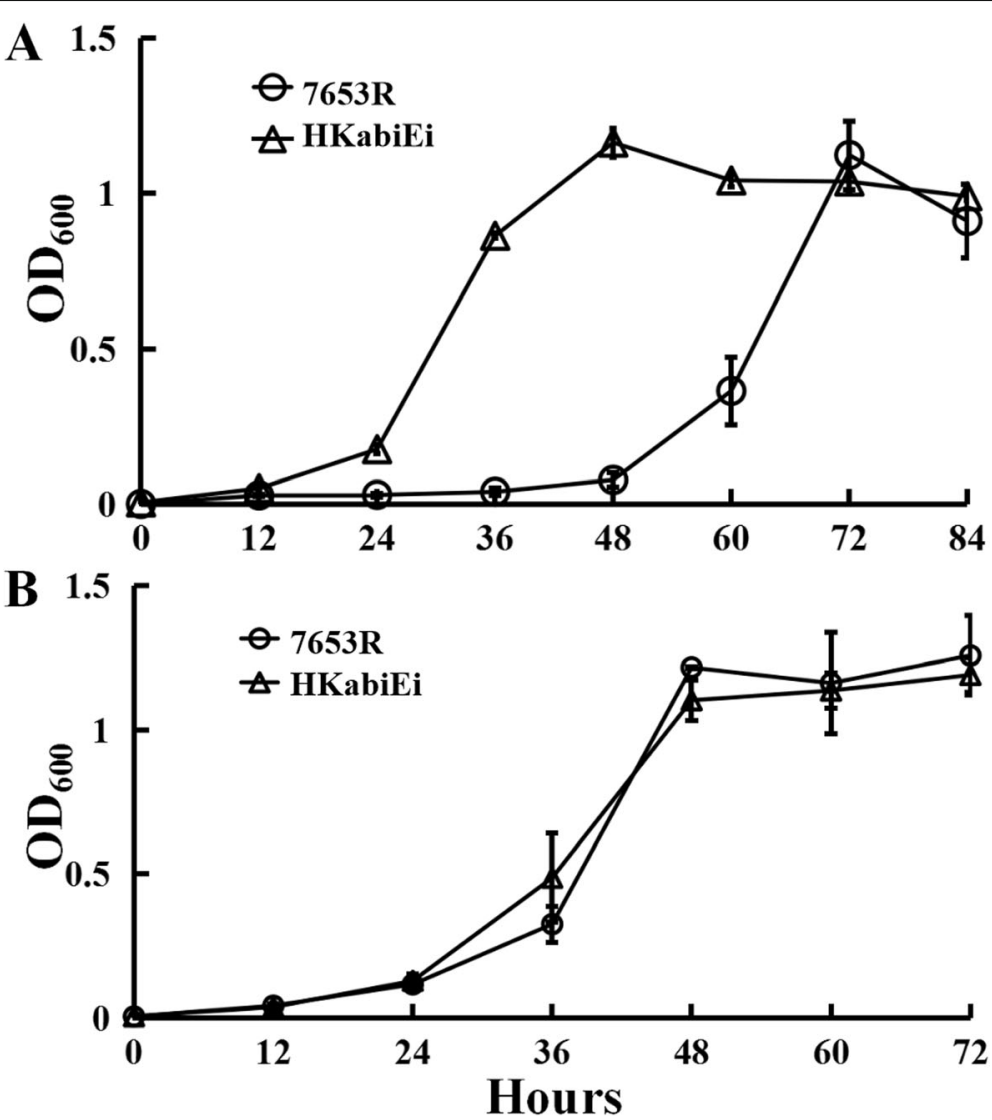

Fig. 3 The effect of antibiotics on bacterial growth. Wild-type 7653R and mutant HKabiEi were grown in AMS Glc/NH4 $4^{+}$supplemented with $2 \mu \mathrm{g}$ $\mathrm{ml}^{-1} \mathrm{Gm}(\mathbf{A})$ or $\mathrm{Cm}(\mathbf{B})$. Data are from three biological samples plus and minus the standard error of the mean ( \pm SEM)

and examined by an Olympus microscope and scanning electron microscopy (SEM). Microscopic analysis of HKabiEi nodules showed that they were small and spherical instead of large and elongated like the wildtype, and compared with wild-type nodules, relatively few nodule cells were infected in the mutant nodules (Fig. 6). SEM analysis demonstrated an aberrant shape of abiEi bacteroids, which were reduced compared to wildtype bacteroids (Fig. 6). A quantitative analysis of bacteroid lengths demonstrated that the mean length of wildtype bacteroids was more than three times those of the abiEi mutant bacteroids, but not different from those of

Table 1 Tolerance of strains to different concentrations of $\mathrm{H}_{2} \mathrm{O}_{2}$

\begin{tabular}{llll}
\hline Strains & \multicolumn{3}{l}{ Diameter(cm) } \\
& \multicolumn{2}{c}{$\left(\mathbf{H}_{\mathbf{2}} \mathbf{O}_{\mathbf{2}}\right) /\left(\mathbf{m m o l} \cdot \mathbf{L}^{-\mathbf{1}}\right)$} & \\
\cline { 2 - 4 } & $\mathbf{2 0}$ & $\mathbf{1 0 0}$ & $\mathbf{2 5 0}$ \\
\hline $7653 R$ & $1.82 \pm 0.08^{\mathrm{a}}$ & $3.03 \pm 0.09^{\mathrm{a}}$ & $3.88 \pm 0.18^{\mathrm{a}}$ \\
HKabiEi & $1.64 \pm 0.09^{\mathrm{b}}$ & $2.60 \pm 0.05^{\mathrm{b}}$ & $3.43 \pm 0.08^{\mathrm{b}}$ \\
HKabiEi(pBBRabiEi) & $2.12 \pm 0.27^{\mathrm{c}}$ & $3.83 \pm 0.12^{\mathrm{c}}$ & $4.28 \pm 0.52^{\mathrm{c}}$ \\
HKabiEi(pBBR1MCS-5) & $1.55 \pm 0.55^{\mathrm{b}}$ & $2.70 \pm 0.30^{\mathrm{b}}$ & $3.45 \pm 0.05^{\mathrm{b}}$
\end{tabular}

The data are the average of at least three replicates. ${ }^{a, b, c}$ values in each column followed by the same letter are not significantly different $(P \leq 0.05)$ the complemented strains HKabiEi(pBBRabiEi). Moreover, the mutant infected nodule cells contained vacuolation and a small number of abnormal bacteroids, with incrassated membrane, scarce content in bacteroids and signs of premature senescence of endosymbiotic bacteria. The results suggested that the mutant-induced nodules were functionally defective.

\section{Activation of abiEi gene in 7653R-inoculated nodules}

As toxin-antitoxin transcriptional regulator AbiEi plays a pivotal role in symbiotic nitrogen fixation, the expression of $a b i E i$ gene in root nodules was analyzed by quantitative RT-PCR (qRT-PCR). The abiEi gene expression was significantly up-regulated in the early stage of nodule formation ( $14 \mathrm{~d}$ ), the nodule maturation stage (28 d) and the late stage (42 d) of nodule development and senescence, and the $a b i E i$ gene had the highest expression level (more than 5 -fold) in nodules at 28 days post inoculation (Fig. 7). Therefore, abiEi gene expression was induced during the symbiotic interaction when compared with free-living cells growing in synthetic medium, and $a b i E i$ may play an important role in 


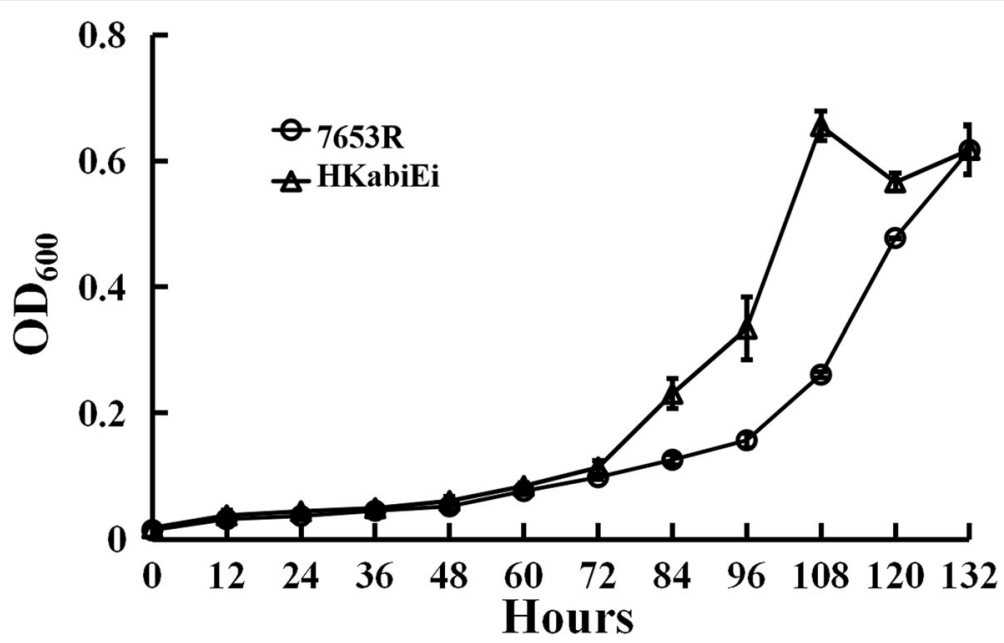

Fig. 4 The effect of SNP on bacterial growth. Wild-type 7653R and mutant HKabiEi were grown in AMS Glc/NH4 ${ }^{+}$supplemented with $10 \mu \mathrm{g} \mathrm{ml}{ }^{-1}$ SNP. Data are from three biological samples plus and minus the standard error of the mean $( \pm$ SEM)

persistence of nodule bacteroids and prevention of premature nodule senescence.

\section{RNA-seq analyses of gene expression in the nodule} bacteroids

RNA-Seq was performed to analyze the overall effect of $a b i E i$ on the transcription pattern of genes in $M$. huakuii bacteroids. A. sinicus root nodules induced by a $M$. huakuii wild-type or an abiEi gene mutant strain were collected at $28 \mathrm{dpi}$ postinoculation. Total RNA was extracted, converted into cDNA libraries, and sequenced using Illumina paired-end sequencing technology. 52million clean sequencing reads were gained from the RNA-seq transcriptomic analysis of the two samples,

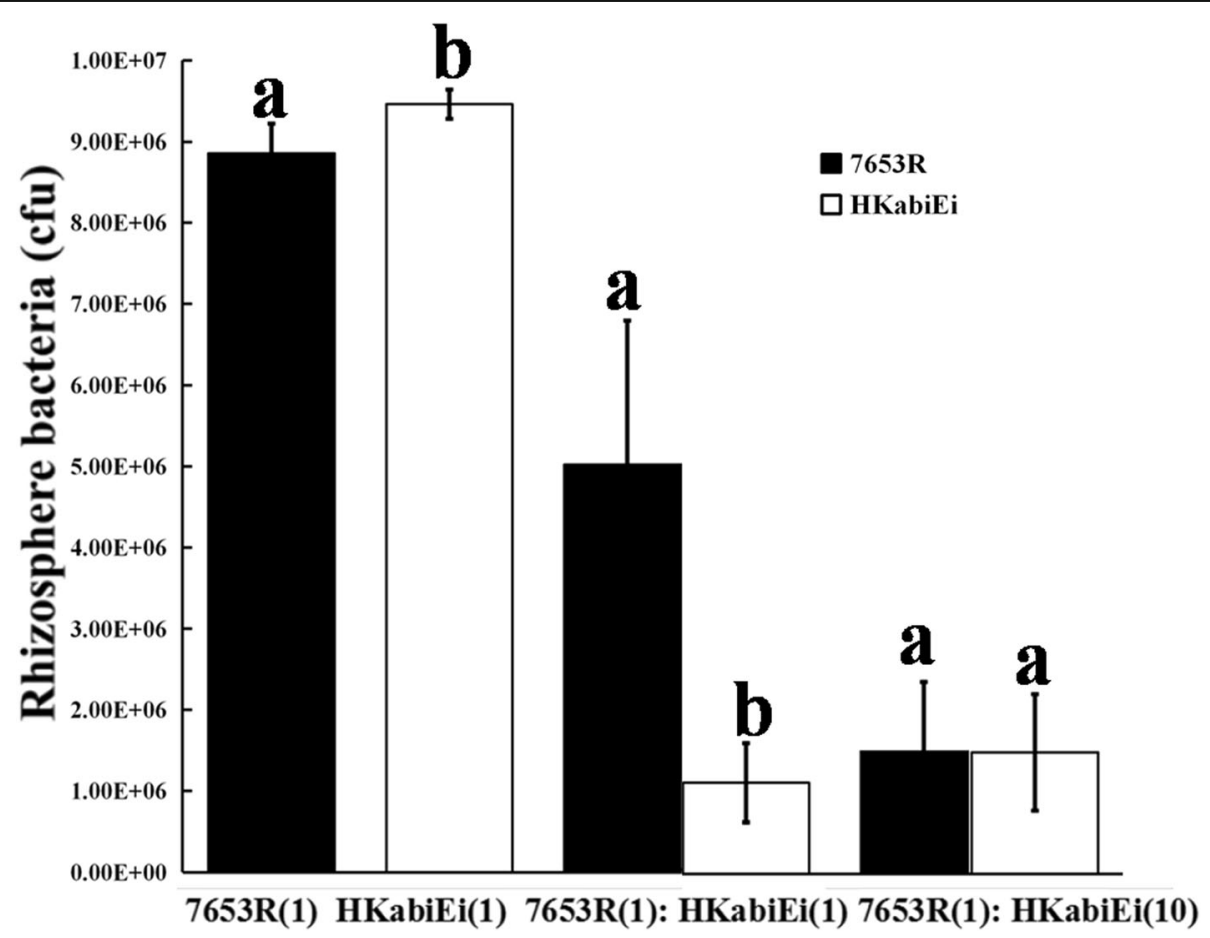

Fig. 5 Bacteria recovered (7 dpi) from the rhizosphere of Astragalus sinicus plants following inoculation with wild-type 7653R and HKabiEi, both individually and together. Inoculation ratios are given on the $x$ axis, with 1 corresponding to $10^{3} \mathrm{CFU}$. Number of bacteria (per plant) recovered from at least 5 plants (mean \pm SEM) are shown. ${ }^{a}$ b Values with different letters are signifificantly different between mutant and wild-type control (two-way ANOVA, $P<0.05$ ) 
Table 2 Symbiotic phenotype of 7653R and HKabiEi ${ }^{a, \beta}$

\begin{tabular}{lllll}
\hline $\begin{array}{l}\text { Strain } \\
\text { M. } \text { huakuii }\end{array}$ & $\begin{array}{l}\text { Plant fresh weight } \\
\text { (mg of plant) }\end{array}$ & $\begin{array}{l}\text { Number of total } \\
\text { nodules per plant }\end{array}$ & $\begin{array}{l}\text { Acetylene reduction activity } \\
\text { (nmol of ethylene/plant/h) }\end{array}$ & $\begin{array}{l}\text { Acetylene reduction activity } \\
\text { (nmol of ethylene/nodule/h) }\end{array}$ \\
\hline $7653 \mathrm{R}$ & $106.05 \pm 8.59^{\mathrm{a}}$ & $17.50 \pm 2.12^{\mathrm{a}}$ & $35.20 \pm 3.68^{\mathrm{a}}$ & $2.01 \pm 0.034^{\mathrm{a}}$ \\
HKabiEi & $63.58 \pm 3.94^{\mathrm{b}}$ & $15.40 \pm 4.39^{\mathrm{a}}$ & $0.90 \pm 0.12^{\mathrm{b}}$ & $0.06 \pm 0.07^{\mathrm{b}}$ \\
HKabiEi(pBBRabiEi) $^{\mathrm{b}}$ & $93.58 \pm 0.83^{\mathrm{a}}$ & $7.00 \pm 1.00^{\mathrm{b}}$ & $15.03 \pm 0.42^{\mathrm{c}}$ & $2.17 \pm 0.66^{\mathrm{a}}$ \\
Control $^{\mathrm{r}}$ & $54.84 \pm .10^{\mathrm{b}}$ & 0 & 0 & 0 \\
\hline
\end{tabular}

${ }^{a}$ Data are the average of at least 5 replicates. Acetylene reduction activity of nodules induced by abiE mutant strain HKabiEi or complementary strain HKabiEi(pBBRabiEi) was compared to that of nodules induced by the wild-type strain $7653 \mathrm{R}$

$\beta$ a,b Values in each column followed by the same letter are not significantly different $(P \leq 0.05)$

${ }^{\gamma}$ Control: plants not inoculated with rhizobial strain

with an average of 26-million reads per sample. In total, 6590 expressed genes were detected by RNA-seq during the symbiosis between the strains and the $A$. sinicus. Gene expression level comparison analysis found that 116 genes were differentially expressed ( $p$ value $\leq 0.001$, with $\log _{2}(\mathrm{FC}) \geq 3$ and $\leq-3$ ), of which 5 were up-regulated and 111 were down-regulated (Tables 3 and 4). We validated the RNA-seq results by RT-PCR using several down-regulated genes as representatives. The datas showed that their expression was significantly decreased in mutant 28-day-old nodules compared to wildtype, which is consistent with the RNA-Seq data (Table 4). Among these differentially expressed genes, $23(19.8 \%)$ were located on the chromosome, 54 (46.6\%) were located on the plasmid pMHa, and 39 (33.6\%) were located on the symbiotic plasmid $\mathrm{pMHb}$, which contains nodulation genes (nod) as well as genes involved in nitrogen fixation (nif and fix). It is worth mentioning that all of the upregulated genes were located on the chromosome,

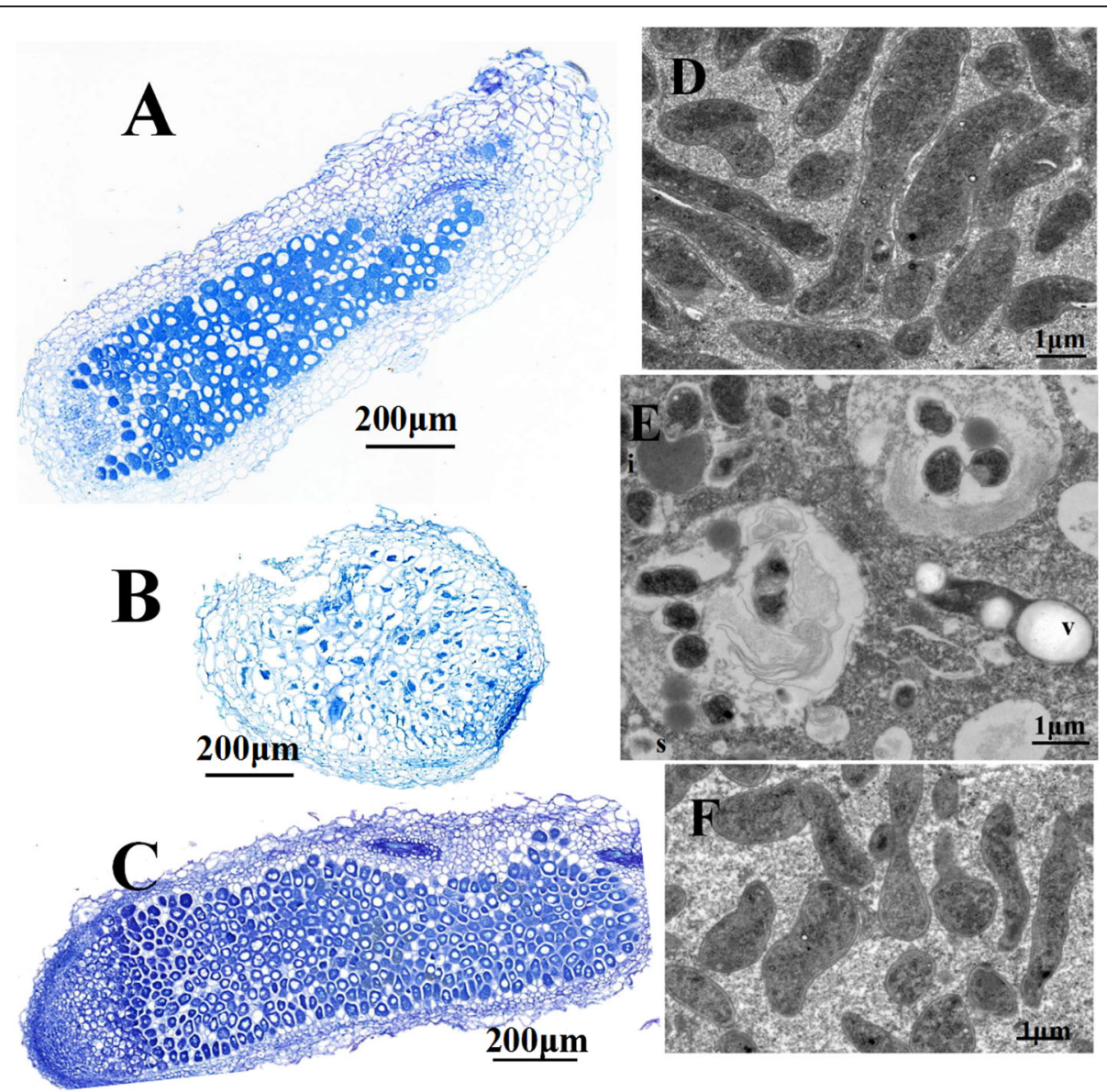

Fig. 6 Structure of 4-week-old Astragalus sinicus nodules and bacteroids. Nodules were induced by M. huakuii 7653R (A, D), HKabiEi (B, E), HKabiEi(pBBRabiEi) (C, F). Scale bars = $200 \mu \mathrm{m}$ (A, B, C), 2 mm (D, E, F). i, incrassated membrane; dissociation; v, vacuolation; s, senescing bacteroid 


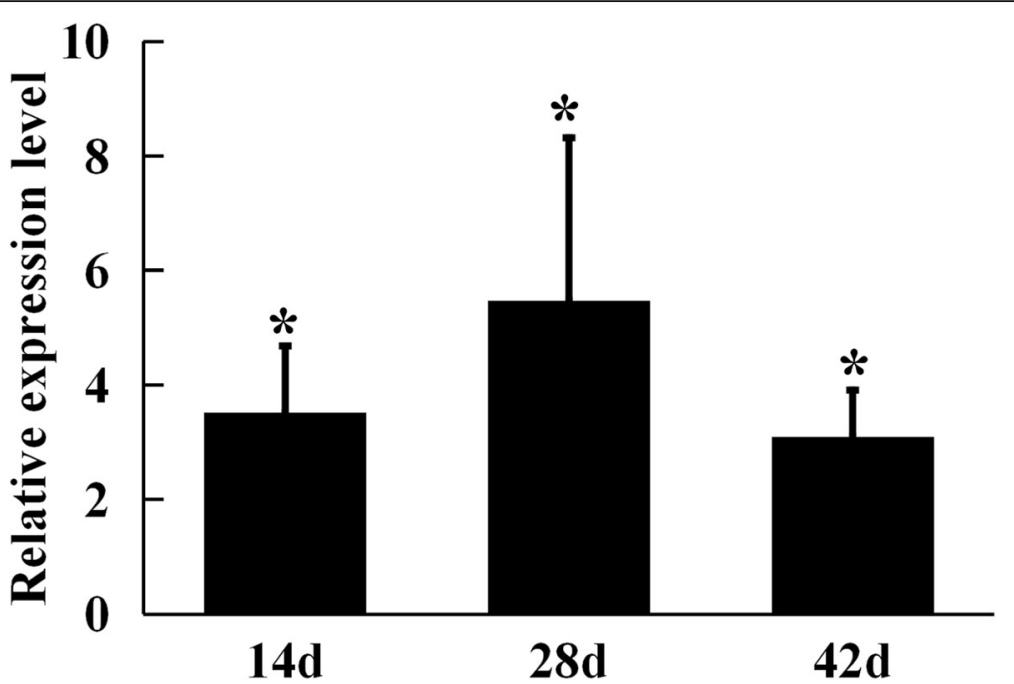

Fig. 7 Expression patterns of abiEi gene in symbiotic nodules. Gene expression levels were examined by real-time RT-PCR. Nodules were collected on different days after inoculation with M. huakuii 7653R. Relative expression of abiEi gene involved in nodule bacteroids compared with $7653 \mathrm{R}$ cells growth in AMS medium. Data are the average of three independent biological samples (each with three technical replicates). Asterisk $\left(^{*}\right)$ indicates a significant difference $(F C>2, P<0.05)$

while more than $80 \%$ of the down-regulated genes were located on the extrachromosomal plasmids.

Among the five up-regulated genes, one encodes type IVb pilin, one codes FMN transferase, one encodes cytochrome b561, and two encode proteins of unknown function. To categorize these differences into modules of biological relevance, the 111 down-regulated genes were annotated. They were functionally classified into 6 categories, which were involved in symbiotic $\mathrm{N}_{2}$-fixation and nitrogen mechanism $(n=21,18.9 \%)$, type VI secretion system $(n=12,10.8 \%)$, electron transport and antioxidant system $(n=16,9.4 \%)$, nucleic acid metabolism $(n=27,24.3 \%)$, Carbon mechanism $(n=11,9.9 \%)$, and hypothetical protein $(n=16,21.6 \%)$ (Table 4$)$. In particular, one fix gene and seven nif genes were required for induction of nitrogen-fixing nodules on $A$. sinicus, and T6SS is rhizobial protein injection machinery with a positive role in Rhizobium-legume symbiosis [23]. The number of differentially expressed symbiosis-associated genes indicated that regulator $\mathrm{AbiEi}$ affects the transcription of a wide range of genes involved in the legume-rhizobium interactions. Further analysis of the differentially expressed genes identified a subset involved in electron transport and antioxidant system. The number of affected the expression of genes involved in the electron transport chain and antioxidant responses also suggested that regulator AbiEi plays an important role in antioxidant systems and the regulation of the electron transport chain. Moreover, 12 genes of T6SS were also found to be significantly over-represented. The genome of many rhizobia encodes T6SS but their role in symbiosis is mostly unknown. However, a functional T6SS, with an effect on symbiosis, has only been shown in $R$. leguminosarum [24]. Furthermore, 27 genes associated with nucleic acid metabolism were found among the 111 genes showing decreased expression in mutant nodules: 10 coding for transposases, six coding for transcriptional or response regulator. Previous studies suggested that transposases (or insertion sequence), transcriptional or response regulator are linked with functions to rhizobial nodulation [25-29]. In addition, 10 carbon mechanism genes and 24 genes of unknown function were found to

Table 3 List of 5 genes that showed significantly increased expression in Astragalus sinicus nodules, induced by the abiEi mutant strain compared to the wild-type 7653R

\begin{tabular}{|c|c|c|c|c|}
\hline Gene ID & Location & Description & $\mathrm{FC}^{\mathrm{a}}$ & $P$ value \\
\hline MCHK_RS00055 & Chro & type IVb pilin & 3.03 & $2.75 \mathrm{E}-05$ \\
\hline MCHK_RS17855 & Chro & flavin mononucleotide transferase & 3.29 & $3.71 \mathrm{E}-08$ \\
\hline MCHK_RS11320 & Chro & hypothetical protein & 3.53 & 2.43E-37 \\
\hline MCHK_RS11325 & Chro & cytochrome b561 & 3.84 & 7.20E-27 \\
\hline MCHK_RS18405 & Chro & hypothetical protein & 4.45 & 4.88E-52 \\
\hline
\end{tabular}

\footnotetext{
${ }^{a} \log _{2}$ of the fold change (FC) in expression of A. sinicus nodules induced by an abiE mutant versus the wild-type 7653R. Chro Chromosome
} 
Table 4 List of 111 genes that showed significantly decreased expression in Astragalus sinicus nodules, induced by the abiEi mutant strain compared to the wild-type 7653R

\begin{tabular}{|c|c|c|c|c|}
\hline Gene ID & Location & Description & $\mathrm{FC}^{\mathrm{a}}$ & RT-PCR \\
\hline \multicolumn{5}{|c|}{ Symbiotic nitrogen Fixation and nitrogen mechanism } \\
\hline MCHK_RS32450 & $\mathrm{pMHb}$ & electron transfer flavoprotein FixA & -8.38 & -9.98 \\
\hline MCHK_RS32495 & $\mathrm{pMHb}$ & nitrogen fixation protein NifZ & -8.37 & \\
\hline MCHK_RS32500 & $\mathrm{pMHb}$ & nitrogen fixation protein NifT & -8.64 & \\
\hline MCHK_RS32360 & $\mathrm{pMHb}$ & nitrogen fixation protein NifQ & -7.12 & \\
\hline MCHK_RS31365 & $\mathrm{pMHa}$ & Nif11-like leader peptide family natural product precursor & -6.00 & \\
\hline MCHK_RS31560 & $\mathrm{pMHa}$ & Nif11 family protein & -4.35 & \\
\hline MCHK_RS32300 & $\mathrm{pMHb}$ & nitrogen fixation protein NifX & -4.30 & \\
\hline MCHK_RS32485 & $\mathrm{pMHb}$ & nitrogenase cofactor biosynthesis protein NifB & -4.53 & \\
\hline MCHK_RS32590 & $\mathrm{pMHb}$ & alanine racemase & -4.28 & \\
\hline MCHK_RS32615 & $\mathrm{pMHb}$ & aminotransferase & -4.82 & \\
\hline MCHK_RS34850 & $\mathrm{pMHb}$ & pilus assembly lipoprotein & -3.11 & \\
\hline MCHK_RS31565 & $\mathrm{pMHa}$ & amino acid epimerase & -4.46 & \\
\hline MCHK_RS31130 & $\mathrm{pMHa}$ & $\mathrm{N}$-acetyltransferase & -3.22 & \\
\hline MCHK_RS08325 & Chro & $A B C$ transporter permease & -4.28 & \\
\hline MCHK_RS11590 & Chro & urea $A B C$ transporter substrate-binding protein & -3.40 & \\
\hline MCHK_RS34620 & $\mathrm{pMHa}$ & $A B C$ transporter permease & -3.24 & \\
\hline MCHK_RS08320 & Chro & $A B C$ transporter permease & -3.16 & \\
\hline MCHK_RS08335 & Chro & ABC transporter ATP-binding protein & -5.74 & \\
\hline MCHK_RS08330 & Chro & $A B C$ transporter substrate-binding protein & -5.15 & \\
\hline MCHK_RS07300 & Chro & $A B C$ transporter substrate-binding protein & -3.12 & \\
\hline MCHK_RS11580 & Chro & urea $\mathrm{ABC}$ transporter permease subunit UrtC & -3.11 & \\
\hline \multicolumn{5}{|c|}{ Type VI secretion system } \\
\hline MCHK_RS31520 & $\mathrm{pMHa}$ & type VI secretion system baseplate subunit TssG & -6.17 & \\
\hline MCHK_RS31510 & $\mathrm{pMHa}$ & type VI secretion system baseplate subunit TssE & -6.12 & \\
\hline MCHK_RS31500 & $\mathrm{pMHa}$ & type VI secretion system contractile sheath large subunit & -6.08 & \\
\hline MCHK_RS31530 & $\mathrm{pMHa}$ & type VI secretion system tip protein VgrG & -6.78 & \\
\hline MCHK_RS31415 & $\mathrm{pMHa}$ & type VI secretion system baseplate subunit TssK & -5.84 & \\
\hline MCHK_RS31515 & $\mathrm{pMHa}$ & type VI secretion system baseplate subunit TssF & -7.52 & \\
\hline MCHK_RS31420 & $\mathrm{pMHa}$ & type VI secretion system-associated FHA domain protein TagH & -5.17 & \\
\hline MCHK_RS31490 & $\mathrm{pMHa}$ & type VI secretion system protein TssA & -4.94 & \\
\hline MCHK_RS31495 & $\mathrm{pMHa}$ & type VI secretion system contractile sheath small subunit & -3.14 & \\
\hline MCHK_RS31505 & pMHa & type VI secretion system tube protein $\mathrm{Hcp}$ & -6.97 & \\
\hline MCHK_RS31430 & $\mathrm{pMHa}$ & type VI secretion system membrane subunit TssM & -6.87 & \\
\hline MCHK_RS31425 & $\mathrm{pMHa}$ & type IVNI secretion system protein & -5.73 & \\
\hline \multicolumn{5}{|c|}{ Electron transport and antioxidant system } \\
\hline MCHK_RS34955 & $\mathrm{pMHa}$ & ferredoxin & -5.68 & \\
\hline MCHK_RS32340 & $\mathrm{pMHb}$ & cytochrome P450 & -10.19 & \\
\hline MCHK_RS32465 & $\mathrm{pMHb}$ & ferredoxin family protein & -8.26 & -9.60 \\
\hline MCHK_RS32535 & $\mathrm{pMHb}$ & cytochrome P450 & -5.40 & \\
\hline MCHK_RS32600 & $\mathrm{pMHb}$ & 4Fe4S-binding protein & -3.77 & \\
\hline MCHK_RS31355 & $\mathrm{pMHa}$ & peroxiredoxin & -8.09 & \\
\hline MCHK_RS31120 & $\mathrm{pMHa}$ & FAD-dependent oxidoreductase & -3.91 & \\
\hline
\end{tabular}


Table 4 List of 111 genes that showed significantly decreased expression in Astragalus sinicus nodules, induced by the abiEi mutant strain compared to the wild-type 7653R (Continued)

\begin{tabular}{|c|c|c|c|c|}
\hline Gene ID & Location & Description & $\mathrm{FC}^{\alpha}$ & RT-PCR \\
\hline MCHK_RS32355 & $\mathrm{pMHb}$ & flavin-dependent oxidoreductase & -8.59 & \\
\hline MCHK_RS34760 & $\mathrm{pMHa}$ & cold-shock protein & -6.69 & \\
\hline MCHK_RS32350 & $\mathrm{pMHb}$ & short-chain dehydrogenase & -8.59 & \\
\hline MCHK_RS34755 & $\mathrm{pMHa}$ & cysteine protease avirulence protein AvrRpt2 & -9.06 & \\
\hline MCHK_RS31555 & $\mathrm{pMHa}$ & L-ascorbate oxidase & -9.55 & -13.04 \\
\hline MCHK_RS08620 & Chro & FAD-binding oxidoreductase & -3.14 & \\
\hline MCHK_RS32460 & $\mathrm{pMHb}$ & FAD-dependent oxidoreductase & -6.41 & \\
\hline MCHK_RS32390 & $\mathrm{pMHb}$ & cold-shock protein & -6.31 & \\
\hline MCHK_RS31275 & $\mathrm{pMHa}$ & NADPH-dependent F420 reductase & -7.42 & \\
\hline \multicolumn{5}{|c|}{ Nucleic acid metabolism } \\
\hline MCHK_RS31350 & $\mathrm{pMHa}$ & recombinase family protein & -3.09 & \\
\hline MCHK_RS31480 & $\mathrm{pMHa}$ & DNA-binding response regulator & -4.52 & \\
\hline MCHK_RS32415 & $\mathrm{pMHb}$ & integrase & -4.71 & \\
\hline MCHK_RS31865 & $\mathrm{pMHb}$ & transposase & -4.70 & \\
\hline MCHK_RS34785 & $\mathrm{pMHa}$ & IS110 family transposase & -7.61 & \\
\hline MCHK_RS31270 & $\mathrm{pMHa}$ & IS6 family transposase & -7.60 & \\
\hline MCHK_RS31860 & $\mathrm{pMHb}$ & IS6 family transposase & -4.07 & \\
\hline MCHK_RS33710 & Chro & IS6 family transposase & -3.65 & \\
\hline MCHK_RS34835 & $\mathrm{pMHa}$ & IS6 family transposase & -3.60 & \\
\hline MCHK_RS32210 & $\mathrm{pMHb}$ & IS6 family transposase & -4.68 & \\
\hline MCHK_RS34745 & $\mathrm{pMHa}$ & IS6 family transposase & -4.63 & \\
\hline MCHK_RS31375 & $\mathrm{pMHa}$ & IS6 family transposase & -3.26 & \\
\hline MCHK_RS31385 & $\mathrm{pMHa}$ & IS21 family transposase & -6.61 & \\
\hline MCHK_RS05160 & Chro & translocated repetitive protein & -8.36 & \\
\hline MCHK_RS31125 & $\mathrm{pMHa}$ & maturation protein 1 & -3.03 & \\
\hline MCHK_RS32635 & $\mathrm{pMHb}$ & autoinducer synthase & -5.04 & \\
\hline MCHK_RS31360 & $\mathrm{pMHa}$ & RNA polymerase sigma-54 factor & -7.22 & \\
\hline MCHK_RS31390 & $\mathrm{pMHa}$ & GntR family transcriptional regulator & -6.74 & \\
\hline MCHK_RS32640 & $\mathrm{pMHb}$ & LuxR family transcriptional regulator & -6.49 & \\
\hline MCHK_RS31250 & $\mathrm{pMHa}$ & XRE family transcriptional regulator & -5.93 & \\
\hline MCHK_RS34740 & $\mathrm{pMHa}$ & hypothetical protein(transcriptional regulator) & -7.35 & \\
\hline MCHK_RS32365 & $\mathrm{pMHb}$ & RNA polymerase sigma-54 factor & -5.62 & \\
\hline MCHK_RS34845 & $\mathrm{pMHa}$ & DNA-binding response regulator & -3.47 & \\
\hline MCHK_RS31380 & $\mathrm{pMHa}$ & nuclear transport factor 2 family protein & -8.90 & \\
\hline MCHK_RS32345 & $\mathrm{pMHb}$ & nuclear transport factor 2 family protein & -8.59 & -8.83 \\
\hline MCHK_RS34720 & $\mathrm{pMHa}$ & DDE-type integrase/transposase/recombinas & -6.91 & \\
\hline MCHK_RS31330 & $\mathrm{pMHa}$ & (Nucleotidyl transferase proteins) NTP transferase & -9.47 & -4.57 \\
\hline \multicolumn{5}{|l|}{ Carbon mechanism } \\
\hline MCHK_RS17040 & Chro & formyl transferase & -4.39 & \\
\hline MCHK_RS17020 & Chro & glycosyltransferase & -4.37 & \\
\hline MCHK_RS00340 & Chro & acyltransferase & -3.88 & \\
\hline MCHK_RS08380 & Chro & acyltransferase & -3.19 & \\
\hline MCHK_RS31550 & $\mathrm{pMHa}$ & tyrosinase & -8.35 & \\
\hline
\end{tabular}


Table 4 List of 111 genes that showed significantly decreased expression in Astragalus sinicus nodules, induced by the abiEi mutant strain compared to the wild-type 7653R (Continued)

\begin{tabular}{|c|c|c|c|c|}
\hline Gene ID & Location & Description & $\mathrm{FC}^{\alpha}$ & RT-PCR \\
\hline MCHK_RS32545 & $\mathrm{pMHb}$ & metalloenzyme & -3.22 & \\
\hline MCHK_RS17025 & Chro & glycosyl hydrolase & -3.70 & \\
\hline MCHK_RS32620 & $\mathrm{pMHb}$ & alkaline phosphatase & -4.31 & \\
\hline MCHK_RS32605 & $\mathrm{pMHb}$ & transketolase & -3.46 & \\
\hline MCHK_RS31340 & pMHa & EamA family transporter & -9.09 & -6.79 \\
\hline MCHK_RS31315 & pMHa & EamA family transporter & -5.96 & \\
\hline \multicolumn{5}{|l|}{ Hypothetical protein } \\
\hline MCHK_RS34775 & $\mathrm{pMHa}$ & hypothetical protein & -9.07 & \\
\hline MCHK_RS32295 & $\mathrm{pMHb}$ & hypothetical protein & -9.03 & \\
\hline MCHK_RS33605 & $\mathrm{pMHb}$ & hypothetical protein & -7.97 & \\
\hline MCHK_RS31545 & pMHa & hypothetical protein & -7.62 & \\
\hline MCHK_RS31320 & $\mathrm{pMHa}$ & hypothetical protein & -9.16 & \\
\hline MCHK_RS34780 & pMHa & hypothetical protein & -6.56 & \\
\hline MCHK_RS09340 & Chro & hypothetical protein & -6.25 & \\
\hline MCHK_RS32420 & $\mathrm{pMHb}$ & hypothetical protein & -6.18 & \\
\hline MCHK_RS33530 & Chro & hypothetical protein & -5.37 & \\
\hline MCHK_RS32630 & $\mathrm{pMHb}$ & hypothetical protein & -5.22 & \\
\hline MCHK_RS32550 & $\mathrm{pMHb}$ & hypothetical protein & -5.03 & \\
\hline MCHK_RS34750 & pMHa & hypothetical protein & -4.98 & \\
\hline MCHK_RS32395 & $\mathrm{pMHb}$ & hypothetical protein & -4.90 & \\
\hline MCHK_RS31405 & $\mathrm{pMHa}$ & hypothetical protein & -4.55 & \\
\hline MCHK_RS31285 & $\mathrm{pMHa}$ & hypothetical protein & -4.16 & \\
\hline MCHK_RS31535 & $\mathrm{pMHa}$ & hypothetical protein & -4.12 & \\
\hline MCHK_RS34940 & $\mathrm{pMHb}$ & hypothetical protein & -3.79 & \\
\hline MCHK_RS32575 & $\mathrm{pMHb}$ & hypothetical protein & -3.59 & \\
\hline MCHK_RS09315 & Chro & hypothetical protein & -3.52 & \\
\hline MCHK_RS31115 & pMHa & hypothetical protein & -4.39 & \\
\hline MCHK_RS32680 & $\mathrm{pMHb}$ & hypothetical protein & -3.38 & \\
\hline MCHK_RS32585 & $\mathrm{pMHb}$ & hypothetical protein & -4.70 & \\
\hline MCHK_RS32595 & $\mathrm{pMHb}$ & hypothetical protein & -4.96 & \\
\hline MCHK_RS31435 & $\mathrm{pMHa}$ & hypothetical protein & -6.69 & \\
\hline
\end{tabular}

${ }^{a} \log _{2}$ of the fold change (FC) in expression of $A$. sinicus nodules induced by an abiE mutant (abiE mt nod) versus the wild type 7653R (wt nod); GHMP kinase: the galactokinase, homoserine kinase, mevalonate kinase, and phosphomevalonate kinase; ROK repressor: open reading frame, kinase; MFS: major facilitator superfamily

be significantly down-regulated in the mutant nodules. qRT-PCR was further performed to confirm changes in gene expression determined by RNA-seq. Seven downregulated genes in five different functional categories were significantly less in HKabiEi-induced nodules compared to nodules induced by wild-type. These results are largely consistent with the changes seen in the RNA-seq assay results.

\section{Discussion}

The type IV TA system AbiE was recently reported, and belonged to the poorly characterized but widespread abortive infection/TA family [12]. To date, there is almost no information of rhizobial AbiE TA system. The Mesorhizobium huakuii AbiE system consists of toxin AbiEii and antitoxin AbiEi. Here we examine the antitoxin $\mathrm{AbiEi}$, which is essential for transcriptional repression of the abiE operon. To investigate the mode of action of this antitoxin, we focus on a abiEi mutant strain of $M$. huakuii that is affected with regard to its symbiotic capacity and stress response.

TA systems are key regulators of bacterial persistence, and are linked to many roles in cell physiology, such as plasmid maintenance, stress response, antibiotic 
resistance, virulence and programmed cell death [30]. Our experiments indicated that the M. huakuii AbiE system can work as a functional TA module in the growth, stress response and antibiotic resistance. Firstly, the abiEi mutant showed slight growth inhibition effect when the strains were cultured in nutrient-rich medium. Due to lack of the antitoxin AbiEi, the abiEi mutant can not neutralize the toxin's activity, and therefore, causes growth stasis. This finding is in agreement with two previous studies reporting that overexpression of the toxin gene in Escherichia coli inhibited its growth [13, 31]. Interestingly, the $a b i E i$ mutant showed no significant difference in AMS minimal medium, suggesting that AbiE TA system does not perform growth inhibition when strains grow at low cell density in nutrientlimiting minimal medium. Secondly, the abiEi mutant displayed significant difference on susceptibility of antibiotics tested. Mutantion in abiEi did not affect M. huakuii chloramphenicol resistance, but displayed an enhanced capacity of $M$. huakuii to gentamicin resistance [32]. It has been reported that the activity of TA system may contribute to the maintenance of antibiotic resistance. Thirdly, compared to wild-type, the abiEi mutant showed enhanced resistance to the oxidizing and reducing agents, $\mathrm{H}_{2} \mathrm{O}_{2}$ and SNP. It was recently reported that the production of Reactive Oxygen Species (ROS) is a common mechanism of cell death induced by bactericidal antibiotics [33].

The symbiotic performance of the abiEi mutant was altered at large levels of nodule function and maintenance. We have observed that the M. huakuii abiEi mutant induced the same number of nodules, exhibited a dramatical decrease in the nitrogen-fixing activity of root nodules (reduced by approx. 97\%). In fact, a S. meliloti strain with a mutant antitoxin gene also had a reduced nitrogen-fixing activity, but formed a higher number of root nodules, which suggested that the host plant was starved for nitrogen due to the inefficient nitrogen fixation, thus initiation of additional nodules was permitted on the roots [10]. The reason why the M. huakuii abiEi mutant did not form more nodules may be due to decreased competitive ability. The root nodules induced by antitoxin gene mutant had decreased size, small amounts of infected cells and bacteroid content, and a severe senescent phenotype. These results indicated that inactive antitoxin AbiEi may affect the formation of the symbiotic nodules on the host roots. In fact, it has been reported that mutant in a functional toxin NtrR increased the symbiotic efficiency of S. meliloti [18], whereas a $S$. meliloti strain with a mutant antitoxin gene had a reduced nitrogen-fixing activity [10]. This symbiotic defect of the antitoxin AbiEi insertion mutant can be explained as a result of the disruption of balance between toxin and antitoxin. The abiEi mutant was defective in transcriptional repression of the $a b i E$ operon, and therefore, overexpressed the toxin AbiEii. On the one hand, many of toxins may induce programmed cell death by directly interacting with bacteroids [34]. Actually, the antitoxin was required for maintenance of transcriptional repression throughout nodule development as its expression was consistently significantly upregulated in the nodules. On the other hand, a toxin VapC from the Leptospiral VapBC toxin-antitoxin module was reported to display ribonuclease activity on the initiator tRNA [35]. As a result, overexpressing the toxin AbiEii may result in a high percentage of downregulated genes such as those which are functionally symbiosis related (Table 4).

Previous reports indicated that the Toxin-Antitoxin is a posttranscriptional regulator of metabolic flux, and antitoxin functions as a transcriptional autoregulator [36, 37]. Henceforth, a RNA-seq experiment was performed to compare the transcript profiles between the root bacteroids infected by abiEi mutant and wild-type 7653R. Firstly, lack of $M$. sativa antitoxin protein has been reported to continuously decrease expression of nif genes in the nodules [10], similar result also happened in the abiEi mutant. Lack of the antitoxin AbiEi reduced transcription of 8 nitrogen fixation genes and 13 genes related to nitrogen transport and mechanism. These suggest that antitoxin AbiEi plays a crucial role in legumerhizobial symbiosis by maintaining the level of nitrogen assimilation and mechanism activity. Secondly, Lack of the antitoxin AbiEi reduced transcription of T6SS, which comprises a series of proteins with structural homology to bacteriophage tail proteins and membrane proteins [38]. It has been reported that T6SS plays a major role in mediating interbacterial competition and might contribute to virulence in plant pathogenic bacteria [39], and Rhizobium etli mutants affected in T6SS structural genes produced plants with lower dry weight and smaller nodules [23]. Thirdly, mutation in AbiEi decreased the activity of redox enzymes such as peroxiredoxin, oxidoreductase, dehydrogenase, oxidase and reductase. Those enzymes were able to effectively reduced intracellular ROS production and involved in redox balance and respiration [40]. It has been reported that antioxidant proteins are critical for nodule activity, and a better antioxidant metabolism can lead to delayed senescence of nodules [41-43]. Fourthly, five downregulation genes are involved in electron transfer. It has been reported that nitric oxide (NO) was also found to play a metabolic role in nodule energy metabolism, and electron transfer chains were found to significantly contribute to $\mathrm{NO}$ production in $\mathrm{N}_{2}$-fixing nodules [44]. Fifthly, inactivation of the AbiEi response regulator directly reduced the activation of four transcriptional regulators (MCHK_RS31390, MCHK_RS32640, MCHK_ 
RS31250 and MCHK_RS34740) and two response regulators (MCHK_RS31480 and MCHK_RS34845). Previous studies suggested that many transcriptional or response regulators are linked with functions to rhizobial nodulation [25-28]. Taken together, M. huakuii AbiEi plays an important role in root nodule symbiosis by regulation of nitrogen fixation gene expression, interbacterial competition, redox balance and respiration, bacteroid formation and senescence.

\section{Conclusions}

Bacterial Toxin-antitoxin systems are composed of bicistronic operons encoding a stable toxin that can harm the host cell and its cognate labile antitoxin. The contribution of toxin-antitoxin to symbiosis and stress response was investigated using the M. huakuii abiEi mutant. The results showed the abiEi mutant strain displayed decreased antioxidative capacity and enhanced gentamicin resistance, and was severely impaired in symbiotic nitrogen-fixing capacity. A quantitative RNA-Seq based transcriptomics approach was also applied to reveal the global transcriptomic responses to AbiEi defect in $M$. huakuii bacteroids from $A$. sinicus root nodules. Compared to the 7653R bacteroids, there were 5 genes were up-regulated and 111 genes were down-regulated in HKabiEi bacteroids. This study provided majority of these differentially expressed genes were grouped into 6 categories and a valuable insight into AbiEi-mediated mechanisms during $M$. huakuii-A. sinicus symbiosis. Furthermore, this study has generated an abundant list of transcript from $M$. huakuii which will provide a fundamental basis for future functional genomic research in M. huakuii and other closely related species.

\section{Methods}

\section{Bacterial growth and media}

The host plant is Astragalus sinicus, which is distributed in all provinces of the Yangtze River Basin in China and cultivated all over the country. The strains, plasmids and primers used in this study are listed in Table 5. M. huakuii strains were grown at $28^{\circ} \mathrm{C}$ in either Tryptone Yeast extract (TY) [49] or Acid Minimal Salts medium (AMS) [50] with D-glucose $(10 \mathrm{mM})$ as a carbon source and $\mathrm{NH}_{4} \mathrm{Cl}(10 \mathrm{mM})$ as a nitrogen source. For growth and qRT-PCR experiments, cells were grown in AMS. When required, the following antibiotics were used at the following final concentrations $\left(\mu \mathrm{g} \mathrm{mL}^{-1}\right)$ : Streptomycin (Str), 500; Ampicillin (Amp), 50; Kanamycin (Km), 20, or 50 (for E. coli growth); Neomycin (Neo), 80, or 250 (for making abiEi mutant); Gentamicin (Gm), 20; Spectinomycin (Spe), 100; Tetracycline (Tc), 5. To monitor culture growth, strains were grown at $28^{\circ} \mathrm{C}$ with shaking $(200 \mathrm{rpm})$ in liquid AMS or TY, and culture optical density at $600 \mathrm{~nm}\left(\mathrm{OD}_{600}\right)$ was measured during the culture period. For antibiotic and sodium nitroprusside (SNP) sensitivity assay, gentamicin, chloramphenicol and SNP were added to each AMS medium at a final concentration of $2 \mu \mathrm{g} \mathrm{mL}^{-1}, 2 \mu \mathrm{gmL}^{-1}$, and $10 \mu \mathrm{g} \mathrm{mL}^{-1}$, respectively.

\section{Expression of AbiE system and AbiEii protein in E. coli}

The AbiE system coding sequence of $1.42 \mathrm{~kb}$ was amplified by PCR from 7653R genomic DNA by using primers AbiE_F and AbiE_R, and the AbiEii coding sequence of $0.7 \mathrm{~kb}$ was amplified by using primers AbiE_F and AbiEii_R. PCR products were digested with $\mathrm{BamHI}$ and HindIII and cloned into the pET-28a(+), and the resulting plasmids were designated pETAbiE for AbiE system and pETAbiEii for toxin gene abiEii. The recombinant plasmids were further transformed in expression host $E$. coli BL21(DE3). Transformants obtained were grown in LB supplemented with appropriate antibiotics. The cultures were induced at $\mathrm{OD}_{600} 0.4$ with $1 \mathrm{mM}$ isopropyl bthiogalactopyranoside (IPTG) and samples were collected every hour for $6 \mathrm{~h}$. The experiment was repeated three times.

\section{Construction and complementation of abiEi gene mutant strain of $M$. huakuii 7653R}

A single-crossover integration mutation in abiEi was made in 7653R. Primers abiEiUP and abiEiLW were used to PCR amplify the abiEi region from 7653R genomic DNA, and the $650 \mathrm{bp}$ internal fragment of the $a b i E i$ gene was cloned into the HindIII and $X b a \mathrm{I}$ sites of pK19mob, giving plasmid pKabiEi. Plasmid pKabiEi was transferred from E. coli to $7653 \mathrm{R}$ and recombined into the genomic abiEi region via single crossover to give strain HKabiEi. Insertions into the abiEi gene of strain 7653R were confirmed by colony PCR using the abiEimap primer and a pK19mob-specific primer pK19A or pK19B $[45,49]$.

To complement the abiEi mutant, primers cabiEi_F and cabiEi_R were used to amplify the complete $a b i E i$ gene from $M$. huakuii 7653R genomic DNA. The PCR

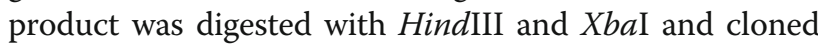
into the broad-host-range vector pBBR1MCS-5, resulting in plasmid pBBRabiEi. Plasmid pBBRabiEi was mated into the mutant strain HKabiEi using the triparental mating method as previously described, while conjugation of HKabiEi with empty vector (pBBR1MCS-5) was used as negative control.

\section{Cellular sensitivity to $\mathrm{H}_{2} \mathrm{O}_{2}$}

Rhizobial cultures were grown in TY medium up to an $\mathrm{OD}_{600}$ of 1 , washed and resuspended in sterilized phosphate buffered saline (PBS) $1 \times(136 \mathrm{mM} \mathrm{NaCl}, 2.6 \mathrm{mM}$ $\left.\mathrm{KCl}, 8.0 \mathrm{mM} \mathrm{Na}_{2} \mathrm{HPO}_{4}, 1.5 \mathrm{mM} \mathrm{KH}_{2} \mathrm{PO}_{4}\right)$. Antioxidation activity were determined by agar diffusion assay using 
Table 5 Strains, plasmids and primers used in this experiment

\begin{tabular}{|c|c|c|}
\hline Strains & Description & Reference, Source, Sequence \\
\hline M. huakii 7653R & Wild type, $\mathrm{Nod}^{+}$on Astragalus sinicus & [45] \\
\hline HKabiEi & 7653R abiEi:pk19mob, Strr $\mathrm{NeO}^{r}$ & This study \\
\hline HKabiEi(pBBRabiEi) & Strain HKabiEi harboring plasmid pBBRabiEi & This study \\
\hline HKabiEi(pBBR1MCS-5) & Strain HKabiEi harboring plasmid pBBR1MCS-5 & This study \\
\hline \multicolumn{3}{|l|}{ Plasmids } \\
\hline pETAbiE & AbiE_F/AbiE_R PCR product in pET-28a(+), $\mathrm{Km}^{r}$ & This study \\
\hline pETAbiEii & AbiE_F/AbiEii_R PCR product in pET-28a(+), $\mathrm{Km}^{r}$ & This study \\
\hline pK19mob & pK19mob pUC19 derivative lacZ mob Km ${ }^{r}$ & [46] \\
\hline pRK2013 & Helper plasmid for mobilizing plasmids $\mathrm{Km}^{r}$ & [47] \\
\hline pKabiEi & abiEiUP/abiEiLW PCR product in pK19mob, $\mathrm{Km}^{r}$ & This study \\
\hline pBBR1MCS-5 & lacPOZ' mob, broad host range, $\mathrm{Gm}^{r}$ & [48] \\
\hline pBBRabiEi & cabiEi_F/cabiEi_R PCR product in pBBR1MCS-5, Gm ${ }^{r}$ & This study \\
\hline \multicolumn{3}{|l|}{ Primer $^{\mathbf{a}}$} \\
\hline AbiE_F & Sense primer used for AbiE system and abiEii gene expression & TTTGGATCCATGTCCTTGGTTGAACCCGA \\
\hline AbiE_R & Antisense primer used for AbiE system expression & $\underline{\text { TITAAGCTTGTGCAGGTCGTAGTAGTGGC }}$ \\
\hline AbiEii_F & $\begin{array}{l}\text { Antisense primer used for MCHK_RS33185 } \\
\text { (abiEii) expression }\end{array}$ & TTIGGATCCGTGAGCACCGACGCCTATCG \\
\hline abiEiUP & $\begin{array}{l}\text { Sense primer for MCHK_RS33180 } \\
\text { (abiEi) mutation }\end{array}$ & TTAAAGCTTGAACCCGACAGCGATCTCCG \\
\hline abiEiLW & Antisense prime for MCHK_RS33180 (abiEi) mutation & TITCTAGAACTTGGCCTCGCTCGATGAG \\
\hline abiEipimap & Mapping PCR primer for abiEi & TGACTCCAGCGCAGCCGTCA \\
\hline cabiei_F & Sense primer for abiEi complementation & TTAAAGCTTGCCACCGACCTTTTATCCTG \\
\hline cabiEi_R & Antisense primer for abiEi complementation & TITCTAGAGCCCTTGAACAGCAGCCGCG \\
\hline pK19A & pK19mob mapping primer & ATCAGATCTTGATCCCCTGC \\
\hline pK19B & pK19mob mapping primer & GCACGAGGGAGCTTCCAGGG \\
\hline Q16SrRNAF & Sense primer for qRT-PCR of 165 rDNA & AACTGAGATGGCTITTGGAG \\
\hline Q16SrRNAR & Antisense primer for qRT-PCR of $16 \mathrm{~S}$ rDNA & GGATGACGTCAAGTCCTCAT \\
\hline Q32450F & Sense primer for qRT-PCR of MCHK_RS32450 & TGGCGAGGTTACCGTACTCA \\
\hline Q32450R & Antisense primer for qRT-PCR of MCHK_RS32450 & AGCGTGTCTGAGCCGGCAAA \\
\hline Q32465F & Sense primer for qRT-PCR of MCHK_RS32465 & GCTCTACCAGAACCGCTATC \\
\hline Q32465R & Antisense primer for qRT-PCR of MCHK_RS32465 & TCGTACAACTCGTAGCATTT \\
\hline Q32345F & Sense primer for qRT-PCR of MCHK_RS32345 & CAACATCCATCAGATCACGA \\
\hline Q32345R & Antisense primer for qRT-PCR of MCHK_RS32345 & TGGCGCATTTCTCCGTCTIT \\
\hline Q31505F & Sense primer for qRT-PCR of MCHK_RS31505 & CAATGGAGTTTCTCAATGGA \\
\hline Q31505R & Antisense primer for qRT-PCR of MCHK_RS31505 & TATAGAGAAGCAGAGGCGTT \\
\hline Q31555F & Sense primer for qRT-PCR of MCHK_RS31555 & TGATCAGGCAGAACCACGGC \\
\hline Q31555R & Antisense primer for qRT-PCR of MCHK_RS31555 & ATCAAGGTCCGTACCCGTTA \\
\hline Q31330F & Sense primer for qRT-PCR of MCHK_RS31330 & TCGGCTCAGAATATCCTCGT \\
\hline Q31330R & Antisense primer for qRT-PCR of MCHK_RS31330 & CGAAATGAAGTCGCTTAGTA \\
\hline Q31340F & Sense primer for qRT-PCR of MCHK_RS31340 & ATTGCCAGCGCCAGCCAGGG \\
\hline Q31340R & Antisense primer for qRT-PCR of MCHK_RS31340 & GGCCGGGCTGCTCTATCTCG \\
\hline QabiEiF & Sense primer for qRT-PCR of abiEi & TCAAGAGCTTCGCCGGATCG \\
\hline QabiEiR & Antisense primer for qRT-PCR of abiEi & ACGGATCACGGCGCGATAGT \\
\hline$\underline{\text { QabiEiif }}$ & Sense primer for qRT-PCR of abiEii & GGCCCGCTCAATGAGTTCCT \\
\hline QabiEiiR & Antisense primer for qRT-PCR of abiEi & TCTGATACCAAAGCAGCAGG \\
\hline
\end{tabular}

${ }^{a}$ Restriction sites in primer sequences are underlined 
filter paper disc diffusion method. Agar plates made from TY were spread with $100 \mu \mathrm{L}$ of bacterial cultures containing $1 \times 10^{9} \mathrm{cfu} \mathrm{mL}^{-1}$. Sterile and dried filter paper discs $6 \mathrm{~mm}$ in diameter, impregnated with different concentrations of $\mathrm{H}_{2} \mathrm{O}_{2}$, were placed on the TY media plate on which bacteria had been spread. Plates were incubated at $28^{\circ} \mathrm{C}$ for $96 \mathrm{~h}$. Results were determined by measuring the diameter of the zones of growth inhibition surrounding the disc. The presence of clear inhibition zones around the bacterial growth indicated the antimicrobial activity. The experiment was repeated at least three times to ensure the reproducibility of the results. The data were analyzed with two-way ANOVA $(P<0.05)$.

\section{Plant experiment and cytological study of nodules}

Astragalus sinicus $L$. was used as a host plant to test nodulation of the $M$. huakuii strains. Seeds were surface-sterilized, placed in $500 \mathrm{~mL}$ pots at two seeds per pot filled with sterile black vermiculite containing nitrogen-free Fahraeus solution. Inoculation with a bacterial suspension of about $10^{8}$ cells/seed was performed on 7-day-old seedlings. The cultivation was carried out in a controlled environment chamber with $16 \mathrm{~h}$ light $/ 8 \mathrm{~h}$ dark period (day/night temperature, $22^{\circ} \mathrm{C}$ and $20^{\circ} \mathrm{C}$ ). Acetylene reduction rate per plant was determined at 31 days postinoculation (dpi) as previously described. The experiment consisted of two independent experiments, each of which had five repeats, and statistical differences were analyzed with one-way ANOVA $(P<0.05)$.

Nodules at $31 \mathrm{dpi}$ were fixed for $12 \mathrm{~h}$ at $4{ }^{\circ} \mathrm{C}$ with $2.5 \%$ glutaraldehyde, rinsed, and post-fixed in $1.5 \%$ phosphate-buffered osmium tetroxide. Ultra-thin sections stained with lead citrate were examined using a Hitachi H-7100 transmission electron microscope. Sections were cut with a microtome and stained with toluidine blue for light microscopy.

\section{Rhizosphere colonization}

Rhizosphere colonization was performed as described previously. Astragalus sinicus seedlings were germinated and grown for 7 days as described above for acetylene reduction activity, and inoculated with $M$. huakuii 7653R and HKabiEi in the cfu ratios 1000:0, 0:1000, 1000:1000 and 1000:10000. Shoots were cut-off after 7 days (14 days after plant), and $10 \mathrm{~mL}$ of sterile phosphate-buffered saline (PBS) buffer (pH 7.4) was added to the roots and vortexed for 15 mins. The samples were further serially diluted and plated on TY agar plates containing either streptomycin and neomycin (only HKabiEi will grow) or streptomycin (both 7653R and HKabiEi will grow), giving the total number of viable rhizosphere- and root-associated bacteria. The ratio of the number of mutant strains to total number of mutant and wild-type strains was calculated for each inoculation test. Each treatment consisted of 10 replications, each of which consisted of a single plant, and statistical differences were analyzed with one-way ANOVA $(P<0.05)$.

\section{RNA isolation and quantitative RT-PCR analysis}

Quantitative real-time reverse transcription PCR (qRTPCR) was performed to determine the abiEi gene expression level in A. sinicus nodules and validate the results of RNA-seq. The total RNA was isolated using Trizol reagent from free-living $M$. huakuii $7653 \mathrm{R}$ or mutant HKabiEi cultivated in AMS liquid medium, or root nodules which were harvested from $A$. sinicus inoculated with strain 7653R or mutant HKabiEi. RNA were reverse transcribed into cDNA using the SuperScript II reverse transcriptase and random hexamers. qRT-PCR analysis was performed using a SYBR Premix ExTaq kit following the manufacturer's instructions on the BIO-RAD CFX96 Real-Time PCR Detection System. The primer sequences are shown in Table 5. The 16S rRNA gene of $M$. huakuii $7653 \mathrm{R}$ was used as a calibrator gene, and the data were obtained. Three independent biological replicates were included in the analysis, and the relative expression levels of the target genes were normalized using the $2^{-\Delta \Delta \mathrm{CT}}$ method.

\section{RNA-seq library preparation and sequencing using the illumina genome analyzer}

At 4 weeks post-inoculation, the nodules of plants inoculated with HKabiEi or 7653R were harvested, immediately frozen in liquid nitrogen and stored at $-80^{\circ} \mathrm{C}$. Total cellular RNA was isolated from frozen nodule tissues using Trizol Reagent (Invitrogen) and RNeasy Mini Kit (Qiagen). Total RNA of each nodule sample was treated with RNase-free DnaseI (TAKARA, Dalian, China), and the absence of contaminating DNA was confirmed by PCR-based assays. Total RNA was assessed using Agilent 2100 Bioanalyzer (Agilent Technologies, Palo Alto, CA, USA), and NanoDrop (Thermo Fisher Scientific Inc). $1 \mu \mathrm{g}$ total RNA with RNA integrity number (RIN) value above 6.5 was used for following library preparation. The rRNA was depleted from total RNA using Ribo-Zero rRNA Removal Kit (Bacteria) (Illumina) [51]. The ribosomal depleted RNA was then fragmented and reverse-transcribed into $\mathrm{cDNA}$ with random primers. The purified double-stranded cDNA by beads was then treated with End Prep Enzyme Mix to repair both ends and add a dA-tailing in one reaction, followed by a T-A ligation to add adaptors to both ends. Next generation sequencing library preparations were constructed according to the manufacturer's protocol. The Qsep100 (Bioptic, Taiwan, China) and Qubit 3.0 
Fluorometer was used to determine the quality of the libraries.

The libraries with different indices were multiplexed and sequenced on an Illumina HiSeq instrument according to manufacturer's instructions (Illumina, San Diego, CA, USA). Sequencing was carried out by Illumina paired-end configuration. The sequencing image processing and base calling were conducted following to Illumina's protocol on the HiSeq instrument. The read length was $90 \mathrm{bp}$, and reads were mapped to the $M$. huakuii genome using Bowtie 2. The HTSeq-count tool was used to generate the total number of uniquely mapped reads for each gene. Three independent biological replicates per sample were processed and sequenced.

\section{Data analysis}

Differences between the average of gene expression for the control and experimental groups were analyzed by the Student's $t$-test using SPSS software, version 18 (SPSS, Inc., Chicago, IL). For the RNA-seq study, the unique reads mapping to the $M$. huakuii genome were used for a differential gene expression analysis using the DESeq2 package. The $P$-values with false discovery rate were adjusted for multiple testing. The false discovery rate $\mathrm{P}$-value $<0.001$ and the absolute value of $\log _{2}(\mathrm{FC}) \geq$ 3 and $\leq-3$ were used to identify statistically significant changes in gene expression. For quantitative RT-PCR analysis, $p<0.05$ was considered to be statistically significant.

\section{Abbreviations}

Abi: Bacterial abortive infection; TA: Toxin-antitoxin; T6SS: Type VI secretion system; qRT-PCR: Quantitative real-time PCR; ROS: Reactive oxygen species; NO: Nitric oxide; SEM: Scanning electron microscopy

\section{Supplementary Information}

The online version contains supplementary material available at https://doi. org/10.1186/s12866-021-02304-0.

Additional file 1: Fig. S1. Inhibition zones of the $\mathrm{H}_{2} \mathrm{O}_{2}$ in disc diffusion test of different strains. Filter paper discs were impregnated with a solution containing $20(A-D), 100(E-H)$ and $250(\mathrm{I}-\mathrm{L}) \mathrm{mg} \mathrm{L}^{-1}$ of $\mathrm{H}_{2} \mathrm{O}_{2}$, and placed on the TY media plate on which bacteria had been spread. Plates were incubated at $28^{\circ} \mathrm{C}$ for 96 h. A, E, I, M. huakuii 7653R; B, F, J, M. huakuii HKabiEi; C, G, K, M. huakuii HKabiEi(pBBRabiEi); D, H, L, M. huakuii HKabiEi(pBBR1MCS-5).

\section{Acknowledgements}

Not applicable.

\section{Authors' contributions}

GC conceived and designed the study. XC, AH, QZ, HW and SL performed the experiments. GC, CY, TL, DH and $X \mathrm{~L}$ analyzed the results, $\mathrm{GC}, \mathrm{XC}$ and $\mathrm{QZ}$ wrote the manuscript. All authors read and approved the final manuscript.

\section{Funding}

This study was supported by the National Natural Science Foundation of China (grant no. 31772399). The funders had no role in design of the study, analysis and interpretation of data and in the preparation of the manuscript.
Availability of data and materials

Raw sequence data from these RNA-seq studies can be accessed via the NCBI Sequence Read Archive, with accession number PRJNA718999.

\section{Declarations}

Ethics approval and consent to participate

Ethical approval according to national legislation (the Law of the People's Republic of China on the Protection of Wildlife) was not necessary as Astragalus sinicus is by far the wide spread green manure of rice field in China, and could improve soil fertility, increase gain yield, benefit nitrogen fixation and energy saving.

\section{Consent for publication}

Not applicable.

\section{Competing interests}

The authors declare that the research was conducted in the absence of any competing interest or commercial or financial relationships that could be construed as a potential conflict of interest.

Received: 4 May 2021 Accepted: 28 August 2021

Published online: 12 September 2021

\section{References}

1. Schuster CF, Bertram R. Toxin-antitoxin systems are ubiquitous and versatile modulators of prokaryotic cell fate. FEMS Microbiol Lett. 2013;340(2):73-85. https://doi.org/10.1111/1574-6968.12074.

2. Melderen LV, Bast M. Bacterial toxin-antitoxin systems: more than selfish entities? PLoS Genet. 2009;5(3):e1000437. https://doi.org/10.1371/journal. pgen.1000437.

3. Wen Z, Wang P, Sun C, Guo Y, Wang X. Interaction of type IV toxin/antitoxin systems in cryptic prophages of Escherichia coli K-12. Toxins (Basel). 2017; 9(3):77. https://doi.org/10.3390/toxins9030077.

4. Maisonneuve E, Shakespeare LJ, Jørgensen MG, Gerdes K. Bacterial persistence by RNA endonucleases. Proc Natl Acad Sci U S A. 2011;108(32): 13206-11. https://doi.org/10.1073/pnas.1100186108.

5. Aizenman E, Engelberg-Kulka H, Glaser G. An Escherichia coli chromosomal "addiction module" regulated by guanosine [corrected] 3',5'bispyrophosphate: a model for programmed bacterial cell death. Proc Natl Acad Sci U S A. 1996;93(12):6059-63. https://doi.org/10.1073/pnas.93.12. 6059.

6. Wang X, Wood TK. Toxin-antitoxin systems influence biofilm and persister cell formation and the general stress response. Appl Environ Microbiol. 2011;77(16):5577-83. https://doi.org/10.1128/AEM.05068-11.

7. Magnuson RD. Hypothetical functions of toxin-antitoxin systems. J Bacteriol. 2007:189(17):6089-92. https://doi.org/10.1128/JB.00958-07.

8. Lobato-Márquez D, Díaz-Orejas R, García-Del Portillo F. Toxin-antitoxins and bacterial virulence. FEMS Microbiol Rev. 2016;40(5):592-609. https://doi.org/1 0.1093/femsre/fuw022

9. Page $\mathrm{R}$, Peti W. Toxin-antitoxin systems in bacterial growth arrest and persistence. Nat Chem Biol. 2016;12(4):208-14. https://doi.org/10.1038/ nchembio.2044.

10. Lipuma J, Cinege G, Bodogai M, Oláh B, Kiers A, Endre G, et al. A vapBCtype toxin-antitoxin module of Sinorhizobium meliloti influences symbiotic efficiency and nodule senescence of Medicago sativa. Environ Microbiol. 2014;16(12):3714-29. https://doi.org/10.1111/1462-2920.12608.

11. Deng YM, Liu CQ, Dunn NW. Genetic organization and functional analysis of a novel phage abortive infection system, AbiL, from Lactococcus lactis. J Biotechnol. 1999;67(2-3):135-49. https://doi.org/10.1016/S0168-1656(98)001 75-8.

12. Hampton HG, Jackson SA, Fagerlund RD, Vogel AIM, Dy RL, Blower TR, et al. AbiEi binds cooperatively to the type IV abiE toxin-antitoxin operator via a positively-charged surface and causes DNA bending and negative autoregulation. J Mol Biol. 2018;430(8):1141-56. https://doi.org/10.1016/j. jmb.2018.02.022.

13. Dy RL, Przybilski R, Semeijn K, Salmond GP, Fineran PC. A widespread bacteriophage abortive infection system functions through a Type IV toxinantitoxin mechanism. Nucleic Acids Res. 2014;42(7):4590-605. https://doi. org/10.1093/nar/gkt1419. 
14. Miklashevichs E, Rohrig H, Schell J, Schmidt J. Perception and signal transduction of rhizobial NOD factors. Crit Rev Plant Sci. 2001;20(4):373-94. https://doi.org/10.1080/20013591099263.

15. Theunis M, Kobayashi H, Broughton WJ, Prinsen E. Flavonoids, NodD1, NodD2, and Nod-box NB15 modulate expression of the y4wEFG locus that is required for indole-3-acetic acid synthesis in Rhizobium sp. strain NGR234. Mol Plant-Microbe Interact. 2004;17(10):1153-61. https://doi.org/10.1094/ MPMI.2004.17.10.1153

16. Miclea PS, Péter M, Végh G, Cinege G, Kiss E, Váró G, et al. Atypical transcriptional regulation and role of a new toxin-antitoxin-like module and its effect on the lipid composition of Bradyrhizobium japonicum. Mol PlantMicrobe Interact. 2010;23(5):638-50. https://doi.org/10.1094/MPMI-23-5-0638.

17. Milunovic B, di Cenzo GC, Morton RA, Finan TM. Cell growth inhibition upon deletion of four toxin-antitoxin loci from the megaplasmids of Sinorhizobium meliloti. J Bacteriol. 2014;196(4):811-24. https://doi.org/10.1128/JB.01104-13.

18. Oláh B, Kiss E, Györgypál Z, Borzi J, Cinege G, Csanádi G, et al. Mutation in the $n t r R$ gene, a member of the vap gene family, increases the symbiotic efficiency of Sinorhizobium meliloti. Mol Plant-Microbe Interact. 2001;14(7): 887-94. https://doi.org/10.1094/MPMI.2001.14.7.887.

19. Fernández-Bachiller MI, Brzozowska I, Odolczyk N, Zielenkiewicz U, Zielenkiewicz P, Rademann J. Mapping protein-protein interactions of the resistance-related bacterial zeta toxin-epsilon antitoxin complex (ع2خ2) with high affinity peptide ligands using fluorescence polarization. Toxins. 2016; 8(7):222. https://doi.org/10.3390/toxins8070222.

20. Park SJ, Son WS, Lee BJ. Structural overview of toxin-antitoxin systems in infectious bacteria: a target for developing antimicrobial agents. Biochim Biophys Acta. 2013;1834(6):1155-67. https://doi.org/10.1016/j.bbapap.2013.02.027.

21. Tang G, Li N, Liu Y, Yu L, Yan J, Luo L. Sinorhizobium meliloti glutathione reductase is required for both redox homeostasis and symbiosis. Appl Environ Microbiol. 2018;84(3):e01937-17. https://doi.org/10.1128/AEM.0193 7-17.

22. Triplett LR, Shidore T, Long J, Miao J, Wu S, Han Q, et al. AvrRxo1 is a bifunctional Type III secreted effector and toxin-antitoxin system component with homologs in diverse environmental contexts. PLoS One. 2016;11(7):e0158856. https://doi.org/10.1371/journal.pone.0158856.

23. Salinero-Lanzarote A, Pacheco-Moreno A, Domingo-Serrano L, Durán D, Ormeño-Orrillo E, Martínez-Romero E, et al. The Type VI secretion system of Rhizobium etli Mim1 has a positive effect in symbiosis. FEMS Microbiol Ecol. 2019;95(5):fiz054. https://doi.org/10.1093/femsec/fiz054.

24. Nelson MS, Sadowsky MJ. Secretion systems and signal exchange between nitrogen-fixing rhizobia and legumes. Front Plant Sci. 2015;6:491. https://doi. org/10.3389/fpls.2015.00491.

25. Loh J, Lohar DP, Andersen B, Stacey G. A two-component regulator mediates population-density-dependent expression of the Bradyrhizobium japonicum nodulation genes. J Bacteriol. 2002;184(6):1759-66. https://doi. org/10.1128/JB.184.6.1759-1766.2002.

26. Janczarek M, Skorupska A. The Rhizobium leguminosarum bv. Trifolii RosR: transcriptional regulator involved in exopolysaccharide production. Mol PlantMicrobe Interact. 2007;20(7):867-81. https://doi.org/10.1094/MPMI-20-7-0867.

27. Del Cerro P, Rolla-Santos AA, Valderrama-Fernández R, Gil-Serrano A, Bellogín RA, Gomes DF, et al. NrcR, a new transcriptional regulator of Rhizobium tropici CIAT 899 involved in the legume root-nodule symbiosis. PLoS One. 2016;11(4):e0154029. https://doi.org/10.1371/journa I.pone.0154029.

28. Bolzan de Campos S, Deakin WJ, Broughton WJ, Passaglia LMP. Roles of flavonoids and the transcriptional regulator Ttsl in the activation of the type III secretion system of Bradyrhizobium elkanii SEMIA587. Microbiology. 2011; 157(3):627-35. https://doi.org/10.1099/mic.0.040873-0

29. Selbitschka W, Zekri S, Schröder G, Pühler A, Toro N. The Sinorhizobium meliloti insertion sequence (IS) elements ISRm102F34-1/ISRm7 and ISRm220-13-5 belong to a new family of insertion sequence elements. FEMS Microbiol Lett. 1999;172(1):1-7. https://doi.org/10.1111/j.1574-6968.1999.tb13441.x.

30. Otsuka Y. Prokaryotic toxin-antitoxin systems: novel regulations of the toxins. Curr Genet. 2016;62(2):379-82. https://doi.org/10.1007/s00294-015-0557-z.

31. Li Z, Song Q, Wang Y, Xiao X, Xu J. Identification of a functional toxinantitoxin system located in the genomic island PYG1 of piezophilic hyperthermophilic archaeon Pyrococcus yayanosii. Extremophiles. 2018;22(3): 347-57. https://doi.org/10.1007/s00792-018-1002-2.

32. Wozniak RA, Waldor MK. A toxin-antitoxin system promotes the maintenance of an integrative conjugative element. PLoS Genet. 2009;5(3): e1000439. https://doi.org/10.1371/journal.pgen.1000439.
33. Kolodkin-Gal I, Sat B, Keshet A, Engelberg-Kulka H. The communication factor EDF and the toxin-antitoxin module mazEF determine the mode of action of antibiotics. PLoS Biol. 2008;6(12):e319. https://doi.org/10.1371/ journal.pbio.0060319.

34. Hazan R, Sat B, Engelberg-Kulka H. Escherichia coli mazEF-mediated cell death is triggered by various stressful conditions. J Bacteriol. 2004;186(11): 3663-9. https://doi.org/10.1128/JB.186.11.3663-3669.2004

35. Lopes AP, Lopes LM, Fraga TR, Chura-Chambi RM, Sanson AL, Cheng E, et al. VapC from the leptospiral VapBC toxin-antitoxin module displays ribonuclease activity on the initiator tRNA. PLoS One. 2014;9(7):e101678. https://doi.org/10.1371/journal.pone.0101678.

36. McKenzie JL, Robson J, Berney M, Smith TC, Ruthe A, Gardner PP, et al. A VapBC toxin-antitoxin module is a posttranscriptional regulator of metabolic flux in mycobacteria. J Bacteriol. 2012;194(9):2189-204. https://doi.org/10.112 8/JB.06790-11.

37. Habib G, Zhu Q, Sun B. Bioinformatics and functional assessment of toxinantitoxin systems in Staphylococcus aureus. Toxins. 2018;10(11):473. https:// doi.org/10.3390/toxins10110473.

38. Gmiter D, Czerwonka G, Kaca WR. Type VB and VI secretion systems as competition agents of gram-negative bacteria[J]. Postep Mikrobiol. 2018; 57(4):360-73. https://doi.org/10.21307/PM-2018.57.4.360.

39. Tian $Y$, Zhao $Y$, Shi L, Cui Z, Hu B, Zhao Y. Type VI secretion systems of Enwinia amylovora contribute to bacterial competition, virulence, and exopolysaccharide production. Phytopathology. 2017;107(6):654-61. https:// doi.org/10.1094/PHYTO-11-16-0393-R.

40. Hu A, Chen X, Luo S, Zou Q, Xie J, He D, et al. Rhizobium leguminosarum glutathione peroxidase is essential for oxidative stress resistance and efficient nodulation. Front Microbiol. 2021;12:627562. https://doi.org/10.33 89/fmicb.2021.627562.

41. Yurgel SN, Simarov BV. Rhizobial electron transport chain and its genetic determinants. Russ J Genet. 1999;35(2):101-9.

42. Becana M, Matamoros MA, Udvardi M, Dalton DA. Recent insights into antioxidant defenses of legume root nodules. New Phytol. 2010;188(4):96076. https://doi.org/10.1111/j.1469-8137.2010.03512.x.

43. Redondo FJ, de la Peña TC, Morcillo CN, Lucas MM, Pueyo JJ. Overexpression of flavodoxin in bacteroids induces changes in antioxidant metabolism leading to delayed senescence and starch accumulation in alfalfa root nodules. Plant Physiol. 2009;149(2):1166-78. https://doi.org/10.11 04/pp.108.129601.

44. Puppo A, Pauly N, Boscari A, Mandon K, Brouquisse R. Hydrogen peroxide and nitric oxide: key regulators of the legume-Rhizobium and mycorrhizal symbioses. Antioxid Redox Signal. 2013;18(16):2202-19. https://doi.org/10.1 089/ars.2012.5136

45. Cheng GJ, Li YG, Zhou JC. Cloning and identification of opa22, a new gene involved in nodule formation by Mesorhizobium huakuii. FEMS Microbiol Lett. 2006;257(1):152-7. https://doi.org/10.1111/j.1574-6968.2006.00158.x.

46. Schäfer A, Tauch A, Jäger W, Kalinowski J, Thierbach G, Pühler A. Small mobilizable multi-purpose cloning vectors derived from the Escherichia coli plasmids pK18 and pK19: selection of defined deletions in the chromosome of Corynebacterium glutamicum. Gene. 1994;145(1):69-73. https://doi.org/1 0.1016/0378-1119(94)90324-7.

47. Figurski DH, Helinski DR. Replication of an origin-containing derivative of plasmid RK2 dependent on a plasmid function provided in trans. Proc Natl Acad Sci U S A. 1979;76(4):1648-52. https://doi.org/10.1073/pnas.76.4.1648.

48. Kovach ME, Phillips RW, Elzer PH, Roop RM 2nd, Peterson KM. pBBR1MCS: a broad-host-range cloning vector. Biotechniques. 1994;16(5):800-2.

49. Zou Q, Zhou Y, Cheng G, Peng Y, Luo S, Wu H, et al. Antioxidant ability of glutaredoxins and their role in symbiotic nitrogen fixation in Rhizobium leguminosarum bv. Viciae 3841. Appl Environ Microbiol. 2020;87(4):e0195620. https://doi.org/10.1128/AEM.01956-20.

50. Cheng G, Karunakaran R, East AK, Munoz-Azcarate O, Poole PS. Glutathione affects the transport activity of Rhizobium leguminosarum 3841 and is essential for efficient nodulation. FEMS Microbiol Lett. 2017;364(8):fnx045. https://doi.org/10.1093/femsle/fnx045.

51. Owens NDL, De Domenico E, Gilchrist MJ. An RNA-Seq protocol for differential expression analysis. Cold Spring Harb Protoc. 2019;2019(6):514. https://doi.org/10.1101/pdb.prot098368.

\section{Publisher's Note}

Springer Nature remains neutral with regard to jurisdictional claims in published maps and institutional affiliations. 